\title{
On the Existence of Pure Nash Equilibria in Weighted Congestion Games
}

\author{
Tobias Harks \\ School of Business and Economics, Maastricht University, Maastricht, The Netherlands, \\ t.harks@maastrichtuniversity.nl \\ Max Klimm \\ Institut für Mathematik, Technische Universität Berlin, 10623 Berlin, Germany, \\ klimm@math.tu-berlin.de
}

\begin{abstract}
We study the existence of pure Nash equilibria in weighted congestion games. Let $\mathscr{b}$ denote a set of cost functions. We say that $\mathscr{b}$ is consistent if every weighted congestion game with cost functions in $\mathscr{C}$ possesses a pure Nash equilibrium. Our main contribution is a complete characterization of consistency of continuous cost functions. We prove that a set $\mathscr{C}$ of continuous functions is consistent for two-player games if and only if $\mathscr{b}$ contains only monotonic functions and for all nonconstant functions $c_{1}, c_{2} \in \mathscr{C}$, there are constants $a, b \in \mathbb{R}$ such that $c_{1}(x)=a c_{2}(x)+b$ for all $x \in \mathbb{R}_{\geq 0}$. For games with at least three players, we prove that $\mathscr{b}$ is consistent if and only if exactly one of the following cases holds: (a) $\mathscr{b}$ contains only affine functions; (b) $\mathscr{C}$ contains only exponential functions such that $c(x)=a_{c} e^{\phi x}+b_{c}$ for some $a_{c}, b_{c}, \phi \in \mathbb{R}$, where $a_{c}$ and $b_{c}$ may depend on $c$, while $\phi$ must be equal for every $c \in \mathscr{C}$. The latter characterization is even valid for three-player games. Finally, we derive several characterizations of consistency of cost functions for games with restricted strategy spaces, such as weighted network congestion games or weighted congestion games with singleton strategies.

Key words: weighted congestion games; pure Nash equilibria; cost functions.

MSC2000 subject classification: Primary: 91A10; secondary: 91A43, 90B10, 68W01

OR/MS subject classification: Primary: games, noncooperative; secondary: mathematics, functions.

History: Received December 7, 2010; revised August 1, 2011, February 1, 2012. Published online in Articles in Advance

May 17, 2012, and updated June 19, 2012.
\end{abstract}

1. Introduction. In many situations, the state of a system is determined by a finite number of independent players, each optimizing an individual objective function. A natural framework for analyzing such decentralized systems are noncooperative games. While it is well known that for finite noncooperative games a Nash equilibrium in mixed strategies always exists, this need not be true for Nash equilibria in pure strategies (PNE for short). One of the fundamental goals in game theory is to characterize conditions under which a Nash equilibrium in pure strategies exists. In this paper, we study this question for weighted congestion games.

Congestion games, as introduced by Rosenthal [32], model the interaction of a finite set of players that compete over a finite set of facilities. A pure strategy of each player is a set of facilities. The cost of facility $f$ is given by a real-valued cost function $c_{f}$ that depends on the number of players using $f$, and the private cost of every player equals the sum of the costs of the facilities in the strategy that she chooses. Rosenthal [32] proved in a seminal paper that such congestion games always admit a PNE by showing that these games possess an exact potential function. In a weighted congestion game, every player has a demand $d_{i} \in \mathbb{R}_{>0}$ that she places on the chosen facilities. The cost of a facility is then a function of the total load on the facility. An important subclass of weighted congestion games are weighted network congestion games. Here, every player is associated with a positive demand that she wants to route from her origin to her destination on a path of minimum cost. In contrast to unweighted congestion games, weighted congestion games do not always admit a PNE. Fotakis et al. [16] and Libman and Orda [24] each constructed a single-commodity network instance with two players having demands one and two, respectively, and showed that these games do not have a PNE. Their instances use different nondecreasing cost values per edge that are defined at the three possible loads, 1,2,3. Goemans et al. [19] constructed a two-player single-commodity instance without a PNE that uses different polynomial cost functions with nonnegative coefficients and degree of at most two. Interestingly, Anshelevich et al. [5] showed that for cost functions of the form $c_{f}(x)=\bar{c}_{f} / x, \bar{c}_{f} \in \mathbb{R}_{\geq 0}$, every two-player game possesses a PNE. For games with affine cost functions, Fotakis et al. [16, 17] proved that every weighted congestion game possesses a PNE. Later, Panagopoulou and Spirakis [30] proved that PNE always exist for instances with uniform exponential cost functions $\left(c_{f}(x)=e^{x}\right)$. Harks et al. [22] generalized this existence result to nonuniform exponential cost functions of the form $c_{f}(x)=a_{f} e^{\phi x}+b_{f}$ for some $a_{f}, b_{f}, \phi \in \mathbb{R}$, where $a_{f}$ and $b_{f}$ may depend on the facility $f$, while $\phi$ must be equal for all facilities. It is worth noting that the positive results of Fotakis et al. [16, 17], Harks et al. [22], and Panagopoulou and Spirakis [30] are particularly important as they establish existence of PNE for the respective sets of cost functions independent of the underlying game structure, that is, independent of the underlying strategy set, demand vector, and number of players, respectively. 
In this paper, we further explore the equilibrium existence problem in weighted congestion games. Our goal is to precisely characterize which types of cost functions actually guarantee the existence of PNE. To formally capture this issue, we introduce the notion of PNE-consistency or simply consistency of a set of cost functions. Let $\mathscr{C}$ be a set of cost functions and let $\mathscr{G}(\mathscr{C})$ be the set of all weighted congestion games with cost functions in $\mathscr{C}$. We say that $\mathscr{C}$ is consistent if every game in $\mathscr{G}(\mathscr{C})$ possesses a PNE. Using this terminology, the results of Fotakis et al. [16, 17], Harks et al. [22], and Panagopoulou and Spirakis [30] yield that $\mathscr{C}$ is consistent if $\mathscr{C}$ contains either affine functions or certain exponential functions. A natural open question is to decide whether there are further consistent functions, that is, functions guaranteeing the existence of a PNE. We thus investigate the following question: How large is the set $\mathscr{C}$ of consistent cost functions? We also introduce a stricter notion of consistency which we term FIP-consistency. Formally, we say that a set $\mathscr{C}$ of cost functions is FIP-consistent, if every game in $\mathscr{G}(\mathscr{C})$ possesses the finite improvement property, that is, every sequence of unilateral improvements is finite; see Monderer and Shapley [28].

1.1. Our results. To obtain a complete characterization of the equilibrium existence problem in weighted congestion games, we first derive necessary conditions. Let $\mathscr{b}$ be a set of continuous functions. We show that if $\mathscr{C}$ is consistent, then $\mathscr{C}$ may only contain monotonic functions. We here use "monotonic" in the literal sense; i.e., every function $c \in \mathscr{C}$ is either nondecreasing or nonincreasing. We further show that monotonicity of cost functions is necessary for consistency even in singleton games with only two players, two facilities, identical cost, functions, and symmetric strategies. As our first main result we show that a set of continuous cost functions $\mathscr{C}$ is consistent for two-player games if and only if $\mathscr{C}$ contains only monotonic functions and for all nonconstant $c_{1}, c_{2} \in \mathscr{C}$, there are constants $a, b \in \mathbb{R}$ such that $c_{1}(x)=a c_{2}(x)+b$ for all $x \in \mathbb{R}_{\geq 0}$. This characterization precisely explains the seeming dichotomy between the positive result of Anshelevich et al. [5] for two-player games and the two-player instances without PNE given by Fotakis et al. [16], Goemans et al. [19], and Libman and Orda [24]. Our second main result establishes a characterization for the general case. We prove that a set $\mathscr{b}$ of continuous functions is consistent for games with at least three players if and only if exactly one of the following cases holds: (a) $\mathscr{C}$ contains only affine functions; (b) $\mathscr{C}$ contains only exponential functions such that $c(x)=a_{c} e^{\phi x}+b_{c}$ for some $a_{c}, b_{c}, \phi \in \mathbb{R}$, where $a_{c}$ and $b_{c}$ may depend on $c$, while $\phi$ must be equal for every $c \in \mathscr{C}$. This characterization is even valid for three-player games. We further show that for both two player games and games with at least three players, consistency of $\mathscr{C}$ is equivalent to FIP-consistency.

While the above characterizations hold for arbitrary strategy spaces, we also study consistency of cost functions for restricted strategy spaces. First, we consider weighted network congestion games. Assuming strictly positive costs, we show that essentially all results translate to directed network congestion games. For games on undirected networks, we give respective characterizations for games with two players and at least four players leaving a gap for three-player games. For singleton weighted congestion games with two players we show that $\mathscr{b}$ is consistent if and only if $\mathscr{b}$ contains only monotonic functions. This characterization does not extend to games with three players. We give an instance with three players and monotonic cost functions without a PNE. For symmetric singleton weighted congestion games, however, we prove that $\mathscr{C}$ is consistent if and only if $\mathscr{C}$ contains only monotonic functions. In contrast to the characterizations for arbitrary strategy spaces, both characterizations do not carry over to FIP-consistency. We provide corresponding instances with improvement cycles.

1.2. Techniques and outline of the paper. The proofs of our main results essentially rely on two ingredients. First, we derive in $\S 3$ for continuous and consistent cost functions two necessary conditions (Monotonicity Lemma (Lemma 3.3) and Extended Monotonicity Lemma (Lemma 3.4)). The Monotonicity Lemma states that any continuous and consistent cost function must be monotonic. The lemma is proved by constructing a generic two-player weighted congestion game in which we identify a unique 4-cycle of deviations of two players. Then, we show that for any nonmonotonic cost function, there is a weighted congestion game with a unique improvement cycle. By adding additional players and carefully choosing the players' weights and strategy spaces, we then derive the Extended Monotonicity Lemma, which ensures that the set of cost functions contained in a certain finite integer linear hull of the considered cost functions must be monotonic. By analyzing functions contained in the finite integer linear hull corresponding to two-player games, we prove in $\S 4$ that a set of continuous cost functions is consistent for two-player games if and only if all cost functions are monotone and every two nonconstant cost functions are affine transformations of each other. In $\S 5$, we consider games with at least three players. We show that the Extended Monotonicity Lemma for games with at least three players implies that consistent and continuous cost functions must be either affine or exponential. In $\S \S 6$ and 7 , we derive characterizations of consistency and FIP-consistency of cost functions for games with restricted strategy spaces, such as weighted network congestion games and weighted singleton congestion games, respectively. 
1.3. Significance. Weighted congestion games are among the core topics in the game theory, operations research, computer science, and economics literature. This class of games has several applications such as scheduling games, routing games, facility location games, network design games, etc; see Ackermann et al. [1], Anshelevich et al. [5], Chen and Roughgarden [11], Gairing et al. [18], Ieong et al. [23], and Milchtaich [27]. In all of the above applications there are two fundamental goals from a system design perspective: (i) the system must be stabilizable, that is, there must exist a stable point (PNE) from which no player wants to unilaterally deviate; (ii) myopic play of the players should guide the system to a stable state. Because the number of players and their types (expressed by the demands and the strategy spaces) are only known to the players and not available to the system designer, it is very natural to study the above two issues with respect to the used cost functions. In fact, in most of the above-mentioned applications, the cost functions are under control of the system designer since they represent the technology associated with the resources, e.g., queuing discipline at routers, latency function in transportation networks, etc. Therefore, our results may help to predict and explain unstable traffic distributions in telecommunication networks and road networks. For instance in telecommunication networks, relevant cost functions are the so-called $M / M / 1$-delay functions (see also Roughgarden and Tardos [35]). These functions are of the form $c_{a}(x)=1 /\left(u_{a}-x\right)$, where $u_{a}$ represents the capacity of arc $a$. In road networks, for instance, the most frequently used functions are monomials of degree 4 put forward by the U.S. Bureau of Public Roads [10]. Our results imply that, for these special types of cost functions, there is always a multi-commodity instance (with three players and identical cost functions) that is unstable in the sense that a PNE does not exist. On the other hand, our characterizations can be used to design a stable system: for instance, uniform $M / M / 1$-delay functions are consistent for two-player games.

Our results are also relevant for the large body of work quantifying the worst-case efficiency loss of PNE for different sets of cost functions; see Awerbuch et al. [6], Christodoulou and Koutsoupias [12], and Aland et al. [3]. While mixed Nash equilibria are guaranteed to exist, their use is often unrealistic in practice. On the other hand, our work reveals that for most classes of cost functions pure Nash equilibria as the stronger solution concept may fail to exist in weighted congestion games. Thus, our work provides additional justification to study the worst-case efficiency loss for different solution concepts, such as sink equilibria (Goemans et al. [19]), correlated and coarse correlated equilibria (Bhawalkar et al. [9], Roughgarden [34]).

1.4. Related work. In contrast to ordinary congestion games as introduced by Rosenthal [32], games with weighted players and/or player-specific cost functions need not possess a PNE. As for weighted players, even two-player games may fail to admit a PNE; see the examples given by Fotakis et al. [16], Goemans et al. [19] and Libman and Orda [24]. Also related is the early work of Rosenthal [33] who showed that in weighted congestion games where players are allowed to split their demand integrally, a PNE need not exist. On the positive side, Fotakis et al. [16] and Panagopoulou and Spirakis [30] proved the existence of a PNE in games with affine and exponential costs, respectively. Dunkel and Schulz [13] showed that it is strongly NP-hard to decide whether or not a weighted congestion game with nonlinear cost functions possesses a PNE. If the strategy of every player contains a single facility only (singleton games), Fotakis et al. [15] showed the existence of PNE for linear cost functions (without a constant). Even-Dar et al. [14] derived the existence of PNE for load balancing games on parallel unrelated machines. Andelman et al. [4] proved even the existence of a strong Nash equilibrium - a strengthening of the pure Nash equilibrium to resilience against coalitional deviations-in scheduling games on unrelated machines. In fact, strong Nash equilibria exist in all singleton weighted congestion games with nondecreasing costs; see Harks et al. [21]. This also holds for nonincreasing cost functions as proven by Rozenfeld and Tennenholtz [36]. Allowing for player-specific cost functions, Milchtaich [25] showed that unweighted singleton congestion games with player-specific cost functions possess at least one PNE. He also presented an instance with weighted players and player-specific cost functions without a PNE. Gairing et al. [18] showed that best response dynamics do not cycle if the player-specific cost functions are linear without a constant term. Milchtaich [27] further showed that general network games with player-specific costs need not admit a PNE in general. In fact, the corresponding decision problem turns out to be NP-complete, as shown by Ackermann and Skopalik [2]. Ieong et al. [23] proved that in congestion games with singleton strategies and nondecreasing cost functions, best response dynamics converge in polynomial time to a PNE. Ackermann et al. [1] extended this result to weighted congestion games with a so-called matroid property, that is, the strategy of every player forms a basis of a matroid. In the same paper, they showed that the matroid property is the maximal property that gives rise to a PNE for all nondecreasing cost functions, that is, for any strategy space not satisfying the matroid property, there is an instance of a weighted congestion game not having a PNE. The consistency approach that we pursue in this paper is orthogonal to that of Ackermann et al. [1]. While they characterize the structure of the strategy space guaranteeing the existence of a PNE assuming arbitrary 
positive and nondecreasing costs, we characterize the structure of cost functions guaranteeing the existence of a PNE assuming arbitrary strategy spaces. Orda et al. [29] study the issue of uniqueness of PNE in weighted network congestion games with splittable demands (see also Bhaskar et al. [8], Milchtaich [26], Richman and Shimkin [31], and Yang and Zhang [37]). They give sufficient conditions for uniqueness of PNE for several classes of cost functions. Interestingly, in the final section of their paper, the authors raise the issue of the existence of pure Nash equilibria in such games (depending on the cost functions) under the assumption that the flow is unsplittable. The results in this paper give a complete answer to their question.

An extended abstract of parts of this paper appeared in the Proceedings of the 37th International Colloquium on Automata, Languages, and Programming, 2010; see Harks and Klimm [20].

2. Preliminaries. We consider finite strategic games $G=(N, S, \pi)$, where $N=\{1, \ldots, n\}$ is the nonempty and finite set of players, $S=\times_{i \in N} S_{i}$ is the nonempty strategy space, and $\pi: S \rightarrow \mathbb{R}^{n}$ is the combined private cost function that assigns a private cost vector $\pi(s)$ to each strategy profile $s \in S$. We consider cost minimization games, and (unless specified otherwise) we allow private cost functions to be negative or positive. We call an element $s \in S$ a strategy profile. For $i \in N$, we write $S_{-i}=\times_{j \neq i} S_{j}$ and $s=\left(s_{i}, s_{-i}\right)$ meaning that $s_{i} \in S_{i}$ and $s_{-i} \in S_{-i}$. A strategy profile $s$ is a pure Nash equilibrium $(P N E)$ if $\pi_{i}(s) \leq \pi_{i}\left(t_{i}, s_{-i}\right)$ for all $i \in N$ and $t_{i} \in S_{i}$. A pair $\left(s,\left(t_{i}, s_{-i}\right)\right) \in S \times S$ is called an improving move (or profitable deviation) of player $i$ if $\pi_{i}\left(s_{i}, s_{-i}\right)>$ $\pi_{i}\left(t_{i}, s_{-i}\right)$. We call a sequence of strategy profiles $\gamma=\left(s^{1}, s^{2}, \ldots\right)$ an improvement path if for every $k$ the tuple $\left(s^{k}, s^{k+1}\right)$ is an improving move for some player $i$. A closed path $\left(s^{1}, \ldots, s^{l}, s^{1}\right)$ is referred to as an l-improvement cycle. A game has the finite improvement property $(F I P)$ if no such cycle exists. A function $P: S \rightarrow \mathbb{R}$ with $P(s)>P(t)$ for all improving moves $(s, t)$ is called a potential function. As noticed by Monderer and Shapley [28], every game that admits a potential function has the FIP and every finite game with the FIP possesses a PNE.

A tuple $\mathbb{M}=\left(N, F, S=\times_{i \in N} S_{i},\left(c_{f}\right)_{f \in F}\right)$ is called a congestion model, where $N$ is the set of players, $F$ is a nonempty, finite set of facilities, and for each player $i \in N$, her collection of pure strategies $S_{i}$ is a nonempty, finite set of subsets of $F$. A cost function $c_{f}: \mathbb{R}_{\geq 0} \rightarrow \mathbb{R}$ is associated with every facility $f \in F$. In contrast to most previous works, we neither assume monotonicity nor positivity of costs. In the following, we define weighted congestion games similar to Goemans et al. [19].

Definition 2.1 (Weighted Congestion Game). Let $\mathbb{M}=\left(N, F, S,\left(c_{f}\right)_{f \in F}\right)$ be a congestion model and $\left(d_{i}\right)_{i \in N}$ be a vector of demands with $d_{i} \in \mathbb{R}_{>0}$. The corresponding weighted congestion game is the strategic game $G(\mathcal{M})=(N, S, \pi)$, where $\pi$ is defined as $\pi=\times_{i \in N} \pi_{i}, \pi_{i}(s)=\sum_{f \in s_{i}} d_{i} c_{f}\left(\ell_{f}(s)\right)$ and $\ell_{f}(s)=\sum_{j \in N: f \in s_{j}} d_{j}$.

We sometimes write $G$ instead of $G(\mathscr{M})$. Let $\mathscr{C}$ be a set of cost functions and let $\mathscr{G}(\mathscr{C})$ be the set of all weighted congestion games with cost functions in $\mathscr{C}$. Then, we say that $\mathscr{C}$ is consistent if every $G \in \mathscr{G}(\mathscr{C})$ admits a PNE; we call $\mathscr{C}$ FIP-consistent if every $G \in \mathscr{G}(\mathscr{C})$ has the FIP. If the set $\mathscr{G}(\mathscr{C})$ is restricted, for instance to two player games etc., we say that $\mathscr{C}$ is consistent for $\mathscr{G}(\mathscr{C})$ if every $G \in \mathscr{G}(\mathscr{C})$ possesses a PNE.

3. Necessary conditions on the existence of a PNE. As a first result, we prove that if $\mathscr{C}$ is consistent, then every function $c \in \mathscr{C}$ is monotonic. We first need a technical lemma.

Lemma 3.1. Let $c: \mathbb{R}_{\geq 0} \rightarrow \mathbb{R}$ be a continuous function. Then, the following two statements are equivalent:

(i) $c$ is monotonic on $\mathbb{R}_{\geq 0}$.

(ii) The following two conditions hold:

(a) For all $x>0$ with $c(x)>c(0)$ there is $\epsilon>0$ such that $c(y) \geq c(x)$ for all $y \in(x, x+\epsilon)$.

(b) For all $x>0$ with $c(x)<c(0)$ there is $\epsilon>0$ such that $c(y) \leq c(x)$ for all $y \in(x, x+\epsilon)$.

Proof. (i) $\Rightarrow$ (ii): Trivial.

For proving (ii) $\Rightarrow$ (i), we first derive a useful property of functions satisfying (ii). Let $c: \mathbb{R}_{\geq 0} \rightarrow \mathbb{R}$ be a continuous function satisfying (ii). Moreover, assume that there is an open interval $(\alpha, \omega)$ with $c(x) \neq c(0)$ for all $x \in(\alpha, \omega)$. We claim that $c$ is nondecreasing on $(\alpha, \omega)$ if $c(x)>c(0)$ for all $x \in(\alpha, \omega)$ and that $c$ is nonincreasing on $(\alpha, \omega)$ if $c(x)<c(0)$ for all $x \in(\alpha, \omega)$. We prove only the first case because the second follows by the same arguments. Let $c(x)>c(0)$ for all $x \in(\alpha, \omega)$. For a contradiction, assume that there are $p_{1}, p_{2} \in(\alpha, \omega)$ with $p_{1}<p_{2}$ and $c\left(p_{1}\right)>c\left(p_{2}\right)$. We define $p_{1}^{\prime}=\max \left\{x \in\left[p_{1}, p_{2}\right]: c(x) \geq c\left(p_{1}\right)\right\}$. Note that the set $\left\{x \in\left[p_{1}, p_{2}\right]: c(x) \geq c\left(p_{1}\right)\right\}$ is nonempty because it contains $p_{1}$ and closed because $c$ is continuous. Using (ii), there is $\epsilon=\epsilon\left(p_{1}^{\prime}\right)>0$ such that $c(y) \geq c\left(p_{1}^{\prime}\right) \geq c\left(p_{1}\right)$ for all $y \in\left(p_{1}^{\prime}, p_{1}^{\prime}+\epsilon\right)$, contradicting the maximality of $p_{1}^{\prime}$.

Now we prove (ii) $\Rightarrow$ (i). Let $\alpha=\inf \{x>0: c(x) \neq c(0)\}$. If $\alpha=\infty$, we are done as $c$ is constant. Otherwise, we claim that $c(x) \neq c(0)$ for all $x>\alpha$. For a contradiction, let $\omega=\min \{x>\alpha: c(x)=c(0)\}$ and 
$\delta=c((\omega+\alpha) / 2)$ (the minimum is attained because $c$ is continuous). By construction, $c(x) \neq c(0)$ for all $x \in(\alpha, \omega)$. If $c(x)>c(0)$ for all $x \in(\alpha, \omega)$, we have $c(x) \geq \delta>c(0)$ for all $x \in((\omega+\alpha) / 2, \omega)$ and thus $c(0)=c(\omega)=\lim _{x>\omega} c(x) \geq \delta>c(0)$, a contradiction. If, on the other hand, $c(x)<c(0)$ for all $x \in(\alpha, \omega)$, we get $c(0)=c(\omega)=\lim _{x{ }_{1} \omega} c(x) \leq \delta<c(0)$, again a contradiction. We conclude that $c(x) \neq c(0)$ for all $x>\alpha$. Thus, for every $\omega>\alpha$, the function $c$ is monotonic on the open interval $(\alpha, \omega)$ and thus, $c$ is monotonic on $\mathbb{R}_{\geq 0}$.

The following existence result for continuous, nonmonotonic functions can be derived directly from Lemma 3.1 and will be very useful in the remainder of this paper.

Lemma 3.2. Let $c: \mathbb{R}_{\geq 0} \rightarrow \mathbb{R}$ be a continuous, nonmonotonic function. Then, there are $x, y \in \mathbb{R}_{>0}$ with $y>x$ such that either $c(y-x)<c(y)<c(x)$ or $c(y-x)>c(y)>c(x)$.

PROOF. Using the characterization of monotonic functions of Lemma 3.1, for every continuous nonmonotonic function $c$, there is $x>0$ such that one of the following holds: $c(x)>c(0)$ and for every $\epsilon>0$ there is $y=y(\epsilon) \in(x, x+\epsilon)$ such that $c(y)<c(x)$; or $c(x)<c(0)$ and for every $\epsilon>0$ there is $y=y(\epsilon) \in(x, x+\epsilon)$ such that $c(y)>c(x)$. Fix such $x$. Because of the continuity of $c$, we have $c(y(\epsilon)-x) \rightarrow c(0)$ and $c(y(\epsilon)) \rightarrow c(x)$ for $\epsilon \rightarrow 0$. For sufficiently small $\epsilon, x$ and $y(\epsilon)$ have the desired property.

The two cases occurring in Lemma 3.2 are depicted in Figure 1. Now consider a facility $f$ with a nonmonotonic cost function and two players with demands $d_{1}=y-x$ and $d_{2}=x$, where $x$ and $y$ are as in Lemma 3.2. Clearly, in case $c(y-x)<c(y)<c(x)$ player 1 prefers to be alone on $f$ while player 2 would like to share the facility with player 1. If $c(y-x)>c(y)>c(x)$, the argumentation works the other way around. This observation is the key to construct a two-player weighted congestion game with singleton strategies that does not admit a PNE.

Lemma 3.3 (Monotonicity Lemma). Let $\mathscr{C}$ be a set of continuous functions. If $\mathscr{C}$ is consistent, then every $c \in C$ is monotonic.

Proof. For a contradiction, suppose that $c \in \mathscr{C}$ is a nonmonotonic function and consider the congestion model $\mathbb{M}=\left(N, F, S,\left(c_{f}\right)_{f \in F}\right)$ with $N=\{1,2\}, F=\{f, g\}, S_{1}=S_{2}=\{\{f\},\{g\}\}, c_{f}=c_{g}=c$. Since $c$ is nonmonotonic, by Lemma 3.2 we can find $x, y \in \mathbb{R}_{>0}$ with $y>x$ such that either $c(y-x)<c(y)<c(x)$ or $c(y-x)>c(y)>c(x)$. Regard the game $G(\mathcal{M})$ with $d_{1}=y-x$ and $d_{2}=x$. Calculating the differences of the deviating players' private costs along the 4-cycle $\gamma=((\{f\},\{f\}),(\{g\},\{f\}),(\{g\},\{g\}),(\{f\},\{g\}),(\{f\},\{f\}))$, we obtain

$$
\begin{gathered}
\pi_{1}(\{g\},\{f\})-\pi_{1}(\{f\},\{f\})=(y-x)(c(y-x)-c(y)), \\
\pi_{1}(\{f\},\{g\})-\pi_{1}(\{g\},\{g\})=(y-x)(c(y-x)-c(x)), \\
\pi_{2}(\{g\},\{g\})-\pi_{2}(\{g\},\{f\})=x(c(y)-c(x)), \quad \pi_{2}(\{f\},\{f\})-\pi_{2}(\{f\},\{g\})=x(c(y)-c(x)) .
\end{gathered}
$$

If $c(y-x)<c(y)<c(x)$, the differences (1)-(2) are negative and $\gamma$ is an improvement cycle. If $c(y-x)>$ $c(y)>c(x)$, we can reverse the direction of $\gamma$ and still get an improvement cycle. Using that every strategy combination is contained in $\gamma$, the claimed result follows.

Besides the continuity of the functions in $\mathscr{C}$, the proof of Lemma 3.3 relies on rather mild assumptions and thus, this result can be strengthened in the following way.
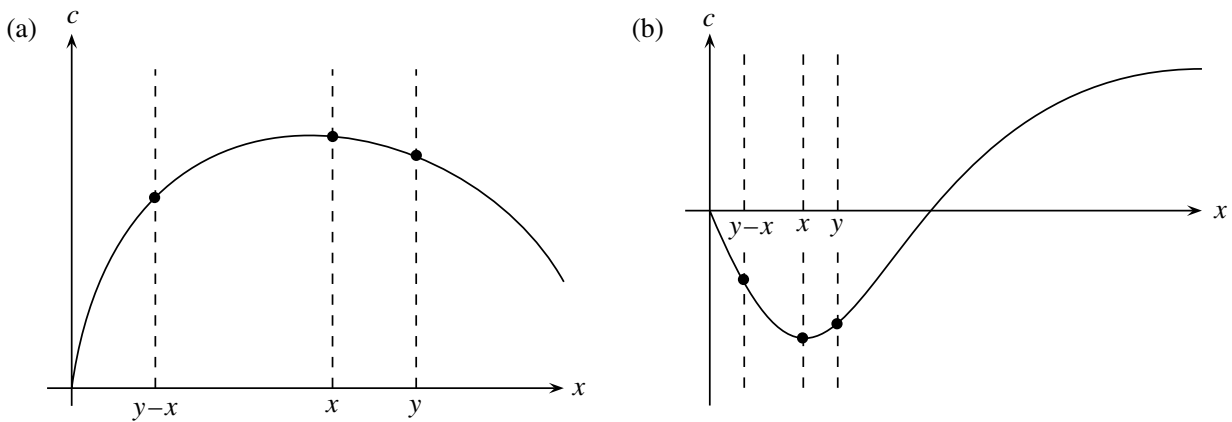

Figure 1. As shown in Lemma 3.2, for every continuous nonmonotonic function there are $x, y \in \mathbb{R}_{>0}$ with $y>x$ such that one of the following cases holds: (a) $c(y-x)<c(y)<c(x)$; (b) $c(y-x)>c(y)>c(x)$. 
Corollary 3.1. Let $\mathscr{C}$ be a set of continuous functions. Let $\mathscr{G}(\mathscr{C})$ be the set of weighted congestion games with cost functions in $\mathscr{b}$ satisfying one or more of the following properties: (i) Each game $G \in \mathscr{G}(\mathscr{b})$ has two players; (ii) Each game $G \in \mathscr{G}(\mathscr{C})$ has two facilities; (iii) For each game $G \in \mathscr{G}(\mathscr{C})$ and each player $i \in N$, the set of her strategies $S_{i}$ contains a single facility only; (iv) Each game $G \in \mathscr{G}(\mathscr{C})$ has symmetric strategies; (v) Cost functions are identical, that is, $c_{f}=c_{g}$ for all $f, g \in F$. If $\mathscr{C}$ is consistent for $\mathscr{G}(\mathscr{b})$, then each $c \in \mathscr{C}$ must be monotonic.

We now extend the Monotonicity Lemma to obtain an even stronger result by regarding more players and more complex strategies. To this end, for $K \in \mathbb{N}$ we consider those functions that can be written as the integral linear combination of $K$ functions in $\mathscr{b}$, possibly with an offset. Formally, we define the finite integer linear hull of $\mathscr{C}$ as

$$
\mathscr{L}_{\mathbb{Z}}(\mathscr{C})=\left\{c: \mathbb{R}_{\geq 0} \rightarrow \mathbb{R}: c(x)=\sum_{k=1}^{K} a_{k} c_{k}\left(x+b_{k}\right): K \in \mathbb{N}, a_{k} \in \mathbb{Z}, b_{k} \in \mathbb{R}_{\geq 0}, c_{k} \in \mathscr{C}\right\},
$$

and show that consistency of $\mathscr{C}$ implies that $\mathscr{L}_{\mathbb{Z}}(\mathscr{C})$ contains only monotonic functions.

Lemma 3.4 (Extended Monotonicity Lemma). Let $\mathscr{b}$ be a set of continuous functions. If $\mathscr{b}$ is consistent, then $\mathscr{L}_{\mathbb{Z}}(\mathscr{C})$ contains only monotonic functions.

Proof. Assume by contradiction that $c \in \mathscr{L}_{\mathbb{Z}}(\mathscr{C})$ is not monotonic. By allowing $c_{k}=c_{l}$ for $k \neq l$, we can omit the integer coefficients $a_{k}$ and rewrite $c$ as $c(x)=\sum_{k=1}^{m_{+}} c_{k}\left(x+b_{k}\right)-\sum_{k=1}^{m_{-}} \bar{c}_{k}\left(x+\bar{b}_{k}\right)$ for some $c_{k}, \bar{c}_{k} \in \mathscr{C}, m_{+}, m_{-} \in \mathbb{N}$, and $b_{k}, \bar{b}_{k} \in \mathbb{R}_{\geq 0}$.

We define the congestion model $M=\left(N, F, S,\left(c_{f}\right)_{f \in F}\right)$, where $N=N_{p} \cup N^{+} \cup N^{-}$and $F=F^{1} \cup F^{2} \cup F^{3} \cup F^{4}$. The set of players $N^{+}$contains for each $c_{k}, 1 \leq k \leq m_{+}$, a player with demand $b_{k}$ and the set of players $N^{-}$ contains for each $\bar{c}_{k}, 1 \leq k \leq m_{-}$, a player with demand $\bar{b}_{k}$. We call the players in $N^{-} \cup N^{+}$offset players. The set $N_{p}=\{1,2\}$ contains two additional (nontrivial) players. Offset players with demand equal to 0 can be removed from the game. For ease of exposition, we assume that all offsets $b_{k}, k=1, \ldots, m_{+}$, and $\bar{b}_{k}, k=1, \ldots, m_{-}$, are strictly positive.

We now explain the strategy spaces and the sets $F^{1}, F^{2}, F^{3}, F^{4}$. For each function $c_{k}, 1 \leq k \leq m_{+}$, we introduce two facilities $f_{k}^{2}, f_{k}^{3}$ with cost function $c_{k}$. For each function $\bar{c}_{k}, 1 \leq k \leq m_{-}$, we introduce two facilities $f_{k}^{1}, f_{k}^{4}$ with cost function $\bar{c}_{k}$. To model the offsets $b_{k}$ in (3), for each offset player $k \in N^{+}$, we define $S_{k}=\left\{f_{k}^{2}, f_{k}^{3}\right\}$. Similarly, for each offset player $k \in N^{-}$, we set $S_{k}=\left\{f_{k}^{1}, f_{k}^{4}\right\}$. The nontrivial players in $N_{p}$ have strategies $S_{1}=\left\{F^{1} \cup F^{2}, F^{3} \cup F^{4}\right\}$ and $S_{2}=\left\{F^{1} \cup F^{3}, F^{2} \cup F^{4}\right\}$, where $F^{1}=\left\{f_{1}^{1}, \ldots, f_{m_{-}}^{1}\right\}, F^{2}=\left\{f_{1}^{2}, \ldots, f_{m_{+}}^{2}\right\}$, $F_{3}=\left\{f_{1}^{3}, \ldots, f_{m_{+}}^{3}\right\}$, and $F^{4}=\left\{f_{1}^{4}, \ldots, f_{m_{-}}^{4}\right\}$.

As $c$ is assumed to be nonmonotonic, by Lemma 3.2, there are $x, y \in \mathbb{R}_{>0}$ with $y>x$ such that either $c(y-x)<c(y)<c(x)$ or $c(y-x)>c(y)>c(x)$. We consider the weighted congestion game $G(M)$ with $d_{1}=y-x$ and $d_{2}=x$ for $1,2 \in N_{p}$. For the 4-cycle

$$
\begin{gathered}
\gamma=\left(\left(F^{1} \cup F^{2}, F^{1} \cup F^{3}, \ldots\right),\left(F^{3} \cup F^{4}, F^{1} \cup F^{3}, \ldots\right),\left(F^{3} \cup F^{4}, F^{2} \cup F^{4}, \ldots\right),\right. \\
\left.\left(F^{1} \cup F^{2}, F^{2} \cup F^{4}, \ldots\right),\left(F^{1} \cup F^{2}, F^{1} \cup F^{3}, \ldots\right)\right),
\end{gathered}
$$

it is straightforward to calculate that

$$
\begin{aligned}
& \pi_{1}\left(F^{3} \cup F^{4}, F^{1} \cup F^{3}, \ldots\right)-\pi_{1}\left(F^{1} \cup F^{2}, F^{1} \cup F^{3}, \ldots\right) \\
& =(y-x)\left(\sum_{k=1}^{m_{+}} c_{k}\left(d_{1}+d_{2}+b_{k}\right)-\sum_{k=1}^{m_{-}} \bar{c}_{k}\left(d_{1}+d_{2}+\bar{b}_{k}\right)+\sum_{k=1}^{m_{-}} \bar{c}_{k}\left(d_{1}+\bar{b}_{k}\right)-\sum_{k=1}^{m_{+}} c_{k}\left(d_{1}+b_{k}\right)\right)=(y-x)(c(y)-c(y-x)), \\
& \pi_{2}\left(F^{3} \cup F^{4}, F^{2} \cup F^{4}, \ldots\right)-\pi_{2}\left(F^{3} \cup F^{4}, F^{1} \cup F^{3}, \ldots\right) \\
& =x\left(-\sum_{k=1}^{m_{+}} c_{k}\left(d_{1}+d_{2}+b_{k}\right)+\sum_{k=1}^{m_{-}} \bar{c}_{k}\left(d_{1}+d_{2}+\bar{b}_{k}\right)+\sum_{k=1}^{m_{+}} c_{k}\left(d_{2}+b_{k}\right)-\sum_{k=1}^{m_{-}} \bar{c}_{k}\left(d_{2}+\bar{b}_{k}\right)\right)=x(c(x)-c(y)), \\
& \pi_{1}\left(F^{1} \cup F^{2}, F^{2} \cup F^{4}, \ldots\right)-\pi_{1}\left(F^{3} \cup F^{4}, F^{2} \cup F^{4}, \ldots\right) \\
& =(y-x)\left(\sum_{k=1}^{m_{+}} c_{k}\left(d_{1}+d_{2}+b_{k}\right)-\sum_{k=1}^{m_{-}} \bar{c}_{k}\left(d_{1}+d_{2}+\bar{b}_{k}\right)+\sum_{k=1}^{m_{-}} \bar{c}_{k}\left(d_{1}+\bar{b}_{k}\right)-\sum_{k=1}^{m_{+}} c_{k}\left(d_{1}+b_{k}\right)\right)=(y-x)(c(y)-c(y-x)), \\
& \pi_{2}\left(F^{1} \cup F^{2}, F^{1} \cup F^{3}, \ldots\right)-\pi_{2}\left(F^{1} \cup F^{2}, F^{2} \cup F^{4}, \ldots\right) \\
& =x\left(-\sum_{k=1}^{m_{+}} c_{k}\left(d_{1}+d_{2}+b_{k}\right)+\sum_{k=1}^{m_{-}} \bar{c}_{k}\left(d_{1}+d_{2}+\bar{b}_{k}\right)-\sum_{k=1}^{m_{-}} \bar{c}_{k}\left(d_{2}+\bar{b}_{k}\right)+\sum_{k=1}^{m_{+}} c_{k}\left(d_{2}+b_{k}\right)\right)=x(c(x)-c(y)) .
\end{aligned}
$$


If $c(y-x)>c(y)>c(x)$, all private cost differences are negative and $\gamma$ is an improvement cycle; if on the other hand $c(y-x)<c(y)<c(x)$, the 4-cycle in the other direction is an improvement cycle. Because every strategy combination is contained in $\gamma$ we get the claimed result.

4. A characterization for two-player games. We analyze implications of the Extended Monotonicity Lemma (Lemma 3.4) for two-player weighted congestion games. First, for ease of exposition, we restrict ourselves to the case $K=2$; that is, we only regard those functions that can be written as an integral linear combination of two functions in $\mathscr{b}$ without offset. We define the following set of functions

$$
\mathscr{L}_{\mathbb{Z}}^{2}(\mathscr{C})=\left\{c: \mathbb{R}_{\geq 0} \rightarrow \mathbb{R}: c(x)=a_{1} c_{1}(x)+a_{2} c_{2}(x), a_{1}, a_{2} \in \mathbb{Z}, c_{1}, c_{2} \in \mathscr{C}\right\} \subseteq \mathscr{L}_{\mathbb{Z}}(\mathscr{C}) .
$$

We remark that by setting all offsets $b_{k}$ in (3) equal to zero, the construction in the proof of Lemma 3.4 only involves two players. Thus, we immediately obtain the following result.

Proposition 4.1. Let $\mathscr{b}$ be a set of continuous functions. If $\mathscr{b}$ is consistent for two-player games, then $\mathscr{L}_{\mathbb{Z}}^{2}(\mathscr{C})$ contains only monotonic functions.

We proceed to investigate sets of functions $\mathscr{C}$ that guarantee that $\mathscr{L}_{\mathbb{Z}}^{2}(\mathscr{C})$ contains only monotonic functions.

Lemma 4.1. Let $c_{1}, c_{2}: \mathbb{R}_{\geq 0} \rightarrow \mathbb{R}$ be two continuous, monotonic, and nonconstant functions. Then, the following are equivalent.

(i) For all $a_{1}, a_{2} \in \mathbb{Z}$ the function $a_{1} c_{1}+a_{2} c_{2}$ is monotonic on $\mathbb{R}_{\geq 0}$.

(ii) There are $a, b \in \mathbb{R}$ such that $c_{2}(x)=a c_{1}(x)+b$ for all $x \geq 0$.

Proof. (ii) $\Rightarrow$ (i): Calculus.

(i) $\Rightarrow$ (ii): Without loss of generality, we may assume that $c_{1}$ and $c_{2}$ are nondecreasing because multiplying functions with -1 has no impact on either statement of the lemma. As $c_{1}$ is nonconstant and nondecreasing, there is $x_{1} \geq 0$ with $c_{1}\left(x_{1}\right)=c_{1}(0)$ and $c_{1}(x)>c_{1}(0)$ for all $x>x_{1}$. Fix such $x>x_{1}$. For all $a_{1}, a_{2} \in \mathbb{Z}$, the function $a_{1} c_{1}+a_{2} c_{2}$ is monotonic. This implies that for every $y>x_{1}$ and every $\alpha \in \mathbb{Q}$ the expressions $\alpha c_{1}(x)+c_{2}(x)-\alpha c_{1}(0)-c_{2}(0)$ and $\alpha c_{1}(y)+c_{2}(y)-\alpha c_{1}(0)-c_{2}(0)$ have identical signs. Thus, for all $y>x_{1}$ and all $\alpha \in \mathbb{Q}$ at least one of the following two cases holds:

(i) $\alpha \geq-\frac{c_{2}(x)-c_{2}(0)}{c_{1}(x)-c_{1}(0)}$ and $\alpha \geq-\frac{c_{2}(y)-c_{2}(0)}{c_{1}(y)-c_{1}(0)}$;

(ii) $\alpha \leq-\frac{c_{2}(x)-c_{2}(0)}{c_{1}(x)-c_{1}(0)}$ and $\alpha \leq-\frac{c_{2}(y)-c_{2}(0)}{c_{1}(y)-c_{1}(0)}$.

This clearly implies

$$
\frac{c_{2}(y)-c_{2}(0)}{c_{1}(y)-c_{1}(0)}=\frac{c_{2}(x)-c_{2}(0)}{c_{1}(x)-c_{1}(0)}
$$

for all $y>x_{1}$, because otherwise any rational

$$
\alpha \in\left(\min \left\{-\frac{c_{2}(y)-c_{2}(0)}{c_{1}(y)-c_{1}(0)},-\frac{c_{2}(x)-c_{2}(0)}{c_{1}(x)-c_{1}(0)}\right\}, \max \left\{-\frac{c_{2}(y)-c_{2}(0)}{c_{1}(y)-c_{1}(0)},-\frac{c_{2}(x)-c_{2}(0)}{c_{1}(x)-c_{1}(0)}\right\}\right)
$$

would violate both constraints. From (4), we obtain

$$
c_{2}(y)=\frac{c_{2}(x)-c_{2}(0)}{c_{1}(x)-c_{1}(0)} \cdot c_{1}(y)-\frac{c_{2}(x)-c_{2}(0)}{c_{1}(x)-c_{1}(0)} \cdot c_{1}(0)+c_{2}(0)
$$

for all $y>x_{1}$. This shows the existence of $a, b \in \mathbb{R}$ with $c_{2}(x)=a c_{1}(x)+b$ for all $x>x_{1}$. Exchanging the roles of $c_{1}$ and $c_{2}$, we may also derive the existence of $a^{\prime}, b^{\prime} \in \mathbb{R}$ such that $c_{1}(x)=a^{\prime} c_{2}(x)+b^{\prime}$ for all $x>x_{2}$, where $x_{2}$ is such that $c_{2}\left(x_{2}\right)=c_{2}(0)$ and $c_{2}(x)>c_{2}(0)$ for all $x>x_{2}$. This implies $x_{1}=x_{2}$. Using the fact that $c_{1}$ and $c_{2}$ are continuous and constant on $\left[0, x_{1}\right]$ finishes the proof.

We are now ready to prove our first main result. 
THEOREM 4.1. Let $\mathscr{C}$ be a set of continuous functions. Let $\mathscr{G}^{2}(\mathscr{C})$ be the set of two-player games such that cost functions are in $\mathscr{C}$. Then, the following conditions are equivalent.

(i) $\mathscr{C}$ is consistent for $\mathscr{G}^{2}(\mathscr{C})$.

(ii) $\mathscr{C}$ is FIP-consistent for $\mathscr{G}^{2}(\mathscr{C})$.

(iii) $\mathscr{C}$ contains only monotonic functions and for all nonconstant $c_{1}, c_{2} \in \mathscr{C}$, there are constants $a, b \in \mathbb{R}$ such that $c_{1}(x)=a c_{2}(x)+b$ for all $x \geq 0$.

Proof. (ii) $\Rightarrow$ (i) is trivial.

(i) $\Rightarrow$ (iii): Using Proposition 4.1 we get that $\mathscr{L}_{\mathbb{Z}}^{2}(\mathscr{C})$ contains only monotonic functions. As $\mathscr{C} \subseteq \mathscr{L}_{\mathbb{Z}}^{2}(\mathscr{C})$, this implies in particular that $\mathscr{C}$ contains only monotonic functions. For all nonconstant functions $c_{1}, c_{2} \in \mathscr{C}$ and all $a_{1}, a_{2} \in \mathbb{Z}$, the function $a_{1} c_{1}+a_{2} c_{2} \in \mathscr{L}_{\mathbb{Z}}^{2}(\mathscr{C})$ is monotonic. Applying Lemma 4.1 then yields the result.

(iii) $\Rightarrow$ (ii): Let $\mathscr{b}$ be as specified in (iii). Trivially, the claimed result holds if $\mathscr{b}$ contains only constant functions. If $\mathscr{C}$ contains a nonconstant function $c$, consider the set $\overline{\mathscr{C}}=\{a c(x)+b: a, b \in \mathbb{R}\} \supseteq \mathscr{C}$. We show that $\overline{\mathscr{C}}$ is consistent for $\mathscr{G}^{2}(\overline{\mathscr{C}})$. To this end, consider an arbitrary two-player game with costs in $\overline{\mathscr{C}}$ and demands $d_{1}<d_{2}$. We distinguish the following three cases.

First case: $c\left(d_{1}\right)<c\left(d_{2}\right)<c\left(d_{1}+d_{2}\right)$, or $c\left(d_{1}\right)>c\left(d_{2}\right)>c\left(d_{1}+d_{2}\right)$. Since $c$ is strictly monotonic with respect to the points $d_{1}, d_{2}$, and $d_{1}+d_{2}$, there is a strictly monotonic function $\tilde{c}$ with $\tilde{c}\left(d_{1}\right)=c\left(d_{1}\right), \tilde{c}\left(d_{2}\right)=$ $c\left(d_{2}\right)$, and $\tilde{c}\left(d_{1}+d_{2}\right)=c\left(d_{1}+d_{2}\right)$. Consequently, we can replace every cost function $c \in \bar{C}=\{a c(x)+b$ : $a, b \in \mathbb{R}\}$ by a cost function $\tilde{c} \in \tilde{\mathscr{C}}=\{a \tilde{c}(x)+b: a, b \in \mathbb{R}\}$ without changing the players' private costs. As shown by Harks et al. [22], for any strictly monotonic function $\tilde{c}$, every weighted congestion game $G$ with two players and cost functions in $\overline{\mathscr{C}}=\{a \tilde{c}(x)+b: a, b \in \mathbb{R}\}$ admits a potential function and, thus, has the FIP.

Second case: $c\left(d_{1}\right)=c\left(d_{2}\right)$. We set $\tilde{d}_{1}=\tilde{d}_{2}=1$ and choose for every facility $f \in F$ a new cost function $\tilde{c}_{f}$ with $\tilde{c}_{f}(1)=c_{f}\left(d_{1}\right)=c_{f}\left(d_{2}\right)$ and $\tilde{c}_{f}(2)=c_{f}\left(d_{1}+d_{2}\right)$. By construction, the unweighted congestion game: with demands $\tilde{d}_{1}, \tilde{d}_{2}$ and costs $\left(\tilde{c}_{f}\right)_{f \in F}$ has the same private costs as the original game. Rosenthal [32] showed the existence of a potential function in all unweighted congestion games; thus, the game has the FIP.

Third case: $c\left(d_{2}\right)=c\left(d_{1}+d_{2}\right)$. We have $\bar{c}\left(d_{2}\right)=\bar{c}\left(d_{1}+d_{2}\right)$ for all $\bar{c} \in \bar{C}$ and thus player 2 is always indifferent whether player 1 shares a facility with her or not. For the FIP and the existence of a PNE, we argue as follows: Consider the vector-valued function $\phi: S \rightarrow \mathbb{R}, s \mapsto\left(\pi_{2}(s), \pi_{1}(s)\right)$ which assigns to every strategy profile the vector which has the private cost of players 2 and 1 in first and second component respectively. We claim that $\phi$ decreases lexicographically along any improvement path. This is trivial for improvement moves of player 2 . Since player 2 is indifferent whether player 1 shares with her a facility or not, every improvement move of player 1 does not affect the private cost of player 2 but decreases the private cost of player 1 . This implies that the lexicographical order of $\phi(s)$ decreases along any improvement path; thus, every such path is finite.

5. A characterization for the general case. We now consider the case $n \geq 3$; that is, we consider weighted congestion games with at least three players. We will show that a set of continuous cost functions is consistent if and only if this set contains either only linear or only certain exponential functions. Our main tool for proving this result is to analyze implications of the Extended Monotonicity Lemma (Lemma 3.4) for three-player weighted congestion games. Formally, define

$$
\mathscr{L}_{\mathbb{Z}}^{3}(\mathscr{C})=\left\{c: \mathbb{R}_{\geq 0} \rightarrow \mathbb{R}: c(x)=a_{1} c_{1}(x)+a_{2} c_{1}(x+\delta): a_{1}, a_{2} \in \mathbb{Z}, c_{1} \in \mathscr{C}, \delta \in \mathbb{R}_{>0}\right\} \subseteq \mathscr{L}_{\mathbb{Z}}(\mathscr{C}) .
$$

Note that $\mathscr{L}_{\mathbb{Z}}^{3}(\mathscr{C})$ involves a single offset $\delta>0$, which requires only three players in the construction of the proof of the Extended Monotonicity Lemma. However, regarding three-player games in which the strategy available to the third player does not intersect with the strategies of the first two players we still get as a necessary condition that $\mathscr{L}_{\mathbb{Z}}^{2}(\mathscr{C})$ may only contain monotonic functions. We, thus, obtain the following result.

Proposition 5.1. Let $\mathscr{C}$ be a set of continuous functions. If $\mathscr{C}$ is consistent for three-player games, then both $\mathscr{L}_{\mathbb{Z}}^{2}(\mathscr{C})$ and $\mathscr{L}_{\mathbb{Z}}^{3}(\mathscr{C})$ contain only monotonic functions.

We proceed to characterize the set of cost functions $\mathscr{C}$ for which $\mathscr{L}_{\mathbb{Z}}^{3}(\mathscr{C})$ contains only monotonic functions.

Lemma 5.1. Let $\mathscr{C}$ be a set of continuous functions. Then, the following two are equivalent:

(i) $\mathscr{L}_{\mathbb{Z}}^{3}(\mathscr{C})$ contains only monotonic functions.

(ii) For every $c \in \mathscr{C}$ either $c(x)=a e^{\phi x}+b$ for some $a, b, \phi \in \mathbb{R}$, or $c(x)=a x+b$ for some $a, b \in \mathbb{R}$. 
Proof. (ii) $\Rightarrow$ (i): Let $c \in \mathscr{C}$ be an exponential or an affine function. By simple calculus one can verify that every function $\tilde{c}(x)=a_{1} c(x)+a_{2} c(x+\delta)$ with $a_{1}, a_{2} \in \mathbb{Z}, \delta \in \mathbb{R}_{>0}$ is exponential if $c$ is exponential and affine if $c$ is affine.

(i) $\Rightarrow$ (ii): By contradiction, assume that $c \in \mathscr{C}$ is neither affine nor exponential. As $\mathscr{L}_{\mathbb{Z}}^{3}(\mathscr{C})$ contains only monotonic functions, for all $\delta>0$ and all $a_{1}, a_{2} \in \mathbb{Z}$ the function $\tilde{c}: \mathbb{R}_{\geq 0} \rightarrow \mathbb{R}, x \mapsto a_{1} c(x)+a_{2} c(x+\delta)$ is monotonic. Referring to Lemma 4.1, this implies that for all $\delta>0$ there are $a, b \in \mathbb{R}$ such that for all $x \geq 0$ :

$$
c(x+\delta)=a c(x)+b .
$$

As $c \in \mathscr{C}$ is neither affine nor exponential on $\mathbb{R}_{\geq 0}$, there must exist four points $0 \leq p_{1}<p_{2}<p_{3}<p_{4}$ following neither an exponential nor an affine law; i.e. there are neither $\alpha, \beta, \phi \in \mathbb{R}$ such that $c\left(p_{i}\right)=\alpha e^{\phi p_{i}}+\beta$ for all $i \in\{1,2,3,4\}$ nor are there $\alpha, \beta \in \mathbb{R}$ such that $c\left(p_{i}\right)=\alpha p_{i}+\beta$ for all $i \in\{1,2,3,4\}$. As $c$ is continuous, we may assume without loss of generality that $p_{1}, p_{2}, p_{3}, p_{4}$ are rational and we write them as $p_{1}=2 m_{1} /(2 k), \ldots, p_{4}=$ $2 m_{4} /(2 k)$ for some $m_{1}, m_{2}, m_{3}, m_{4}, k \in \mathbb{N}$. For $\delta=1 / k$ we derive from (5) that there are $a, b \in \mathbb{R}$ such that for all $n \in \mathbb{N}$ :

$$
\begin{gathered}
c((n+1) / k)=a c(n / k)+b, \\
c((n+2) / k)=a c((n+1) / k)+b .
\end{gathered}
$$

Subtracting (6) from (7) and rearranging terms, we obtain for all $n \in \mathbb{N}$ :

$$
c((n+2) / k)-(a+1) c((n+1) / k)+a c(n / k))=0 .
$$

This defines a second-order linear homogeneous recurrence relation on the sequence $c(n / k)_{n \in \mathbb{N}}$. Such recurrence relations can be solved with the method of characteristic equations; see Balakrishnan [7, §3.2] for more details. The characteristic equation of the recurrence relation equals $x^{2}-(a+1) x+a=(x-1)(x-a)$. If $a \neq 1$, then the characteristic equation has two distinct roots and we obtain for even $m$ that

$$
c(m / k)=\beta \cdot 1^{m}+\alpha \cdot a^{m}=\beta+\alpha \cdot|a|^{m}=\alpha \cdot \exp (m \ln |a|)+\beta
$$

for some constants $\alpha, \beta \in \mathbb{R}$. If on the other hand $a=1$, we can evaluate $c(m / k)$ as

$$
c(m / k)=\beta \cdot 1^{m}+\alpha m \cdot 1^{m}=\alpha \cdot m+\beta
$$

for some constants $\alpha, \beta \in \mathbb{R}$.

We are now ready to state our second main theorem.

THEOREM 5.1. Let $\mathscr{C}$ be a set of continuous functions. Then, the following three are equivalent:

(i) $\mathscr{C}$ is consistent.

(ii) $\mathscr{C}$ is FIP-consistent.

(iii) $\mathscr{C}$ contains only affine functions or $\mathscr{C}$ contains only functions of type $c(x)=a_{c} e^{\phi x}+b_{c}$, where $a_{c}, b_{c} \in \mathbb{R}$ may depend on $c$ while $\phi \in \mathbb{R}$ is independent of $c$.

Proof. (ii) $\Rightarrow$ (i) is trivial.

(iii) $\Rightarrow$ (ii) follows because every weighted congestion games with such cost functions possesses a weighted potential; see Fotakis et al. [16], Harks et al. [22], and Panagopoulou and Spirakis [30].

(i) $\Rightarrow$ (iii): By Proposition 5.1 both $\mathscr{L}_{\mathbb{Z}}^{2}(\mathscr{C})$ and $\mathscr{L}_{\mathbb{Z}}^{3}(\mathscr{C})$ may only contain monotonic functions. Applying Lemma 5.1 we obtain that every $c \in \mathscr{C}$ is either affine or exponential. In addition, as shown in Lemma 4.1 for each two nonconstant functions $c_{1}, c_{2} \in \mathscr{C}$, there are $a, b \in \mathbb{R}$ such that $c_{2}(x)=a c_{1}(x)+b$ for all $x \geq 0$. Both results together imply (iii).

We conclude this section by giving an example that illustrates the main ideas presented so far. Recall, that Theorem 5.1 establishes that for each continuous, nonaffine and nonexponential cost function $c$, there is a weighted congestion game $G$ with uniform cost function $c$ on all facilities that does not admit a PNE. In the following example, we show how such a game for $c(x)=x^{3}$ is constructed.

EXAMPLE 5.1. As the function $c(x)=x^{3}$ is neither affine nor exponential, there are $a_{1}, a_{2} \in \mathbb{Z}$ and $\delta \in \mathbb{R}_{>0}$ such that $\tilde{c}(x)=a_{1} c(x)+a_{2} c(x+\delta)$ has a strict local extremum. In fact, we can choose $a_{1}=2, a_{2}=-1$, and $\delta=1$, that is, the function $\tilde{c}(x)=2 c(x)-c(x+1)=2 x^{3}-(x+1)^{3}$ has a strict local minimum at $x_{0}=1+\sqrt{2}$. In particular, we can choose $d_{1}=1$ and $d_{2}=2$ such that $\tilde{c}\left(d_{1}\right)=-6>\tilde{c}\left(d_{2}\right)=-11<\tilde{c}\left(d_{1}+d_{2}\right)=-10$. The weighted congestion game without PNE is now constructed as follows: We introduce $2\left(\left|a_{1}\right|+\left|a_{2}\right|\right)$ facilities $f_{1}, \ldots, f_{6}$ and the following strategies $s_{1}^{a}=\left\{f_{1}, f_{2}, f_{3}\right\}, s_{1}^{b}=\left\{f_{4}, f_{5}, f_{6}\right\}, s_{2}^{a}=\left\{f_{1}, f_{2}, f_{4}\right\}, s_{2}^{b}=\left\{f_{3}, f_{5}, f_{6}\right\}$, and 
(a) Strategies

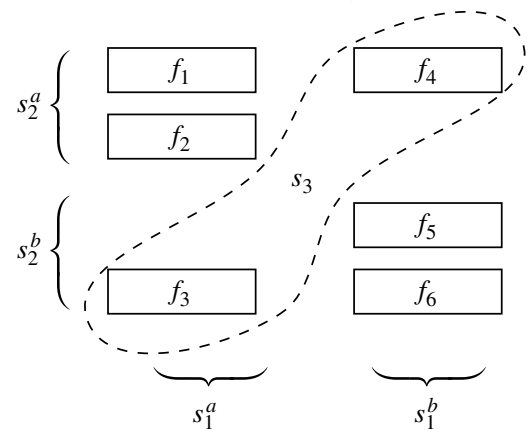

(b) Improvement cycle $\gamma$

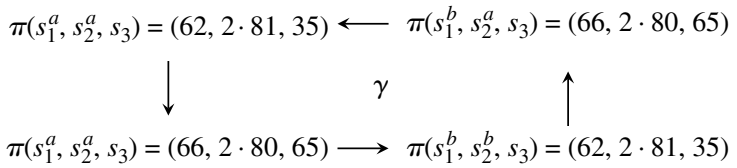

FIGURE 2. (a) The players' strategies and (b) the improvement cycle $\gamma$ of the game constructed in Example 5.1 that does not admit a PNE.

$s_{3}=\left\{f_{3}, f_{4}\right\}$. We then set $S_{1}=\left\{s_{1}^{a}, s_{1}^{b}\right\}, S_{2}=\left\{s_{2}^{a}, s_{2}^{b}\right\}$, and $S_{3}=\left\{s_{3}\right\}$; see Figure 2(a) for an illustration of the strategies. The so-defined game has four strategy profiles, namely $\left(s_{1}^{a}, s_{2}^{a}, s_{3}\right),\left(s_{1}^{a}, s_{2}^{b}, s_{3}\right),\left(s_{1}^{b}, s_{2}^{a}, s_{3}\right),\left(s_{1}^{b}, s_{2}^{b}, s_{3}\right)$. As Player 3 is an offset player, she has a single strategy only; thus, the players' private costs depend only on the choice of players 1 and 2. We derive that the 4-cycle $\gamma$ shown in Figure 2(b) is a best-reply cycle in $G$. As there are no strategy profiles outside $\gamma$ we conclude that $G$ has no PNE.

6. Weighted network congestion games. In this section, we discuss the implications of our characterizations to the important subclass of weighted network congestion games. In these games, the facilities correspond to edges of a directed or undirected graph. Every player is associated with a positive demand that she wants to route from her origin to her destination on a path of minimum cost. We consider directed and undirected networks separately, starting with directed networks.

6.1. Directed networks. We first give a version of the Extended Monotonicity Lemma for directed networks with two players and strictly positive costs.

Lemma 6.1 (Extended Monotonicity Lemma for Two-Player Games on Directed Networks). Let $\mathscr{C}$ be a set of strictly positive and continuous functions. If $\mathscr{C}$ is consistent for two-player directed network congestion games, then $\mathscr{L}_{\mathbb{Z}}^{2}(\mathscr{C})$ contains only monotonic functions.

Proof. Because singleton congestion games are a subclass of directed network congestion games, by Corollary 3.1 every set $\mathscr{b}$ of consistent functions contains only monotonic functions. For a contradiction, assume that there are $a_{1}, a_{2} \in \mathbb{Z}$ and monotonic functions $c_{1}, c_{2} \in \mathscr{C}$ such that the function $c: \mathbb{R}_{\geq 0} \rightarrow \mathbb{R}$ defined as $c(x)=a_{1} c_{1}(x)+a_{2} c_{2}(x)$ is not monotonic. By Lemma 3.2 there are $x, y \in \mathbb{R}_{>0}$ with $y>x$ such that either $c(y-x)<c(y)<c(x)$ or $c(y-x)>c(y)>c(x)$. We choose the demands equal to $d_{1}=y-x$ and $d_{2}=x$. Note that $c$ is monotonic if and only if $-c$ is monotonic; thus we may assume w.l.o.g. that $a_{2}>0$. To define the players' strategies we distinguish the following two cases.

(a) Case $a_{1}<0$

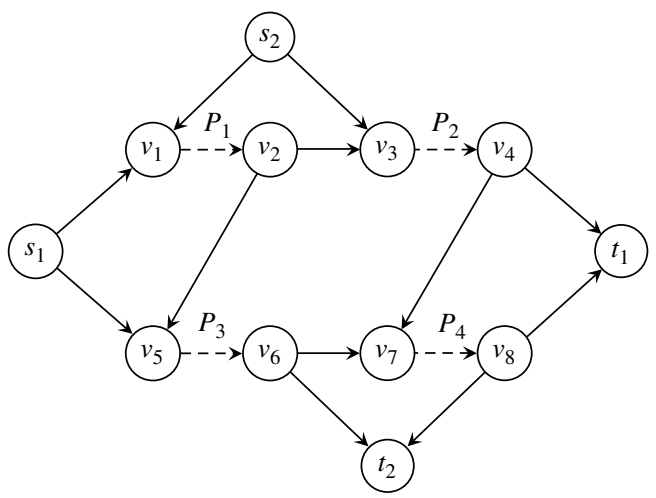

(b) Case $a_{1}>0$

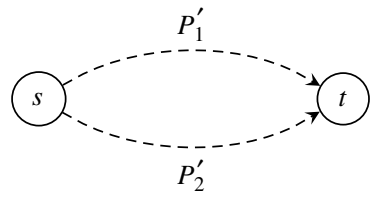

FIGURE 3. Directed network congestion games used in the proof of the Extended Monotonicity Lemma for Two-Player Directed Networks (Lemma 6.1). 
First case: $a_{1}<0$ : We use a construction similar to the proof of Lemma 3.4. To define the players' strategy spaces, consider the network in Figure 3. The two players are represented by the two source-terminal pairs $\left(s_{i}, t_{i}\right), i=1,2$. The set of strategies available to player $i$ equals the set of directed $\left(s_{i}, t_{i}\right)$-paths. The dashed edges in Figure 3(a) correspond to directed paths $P_{1}, \ldots, P_{4}$, which we choose as follows: the directed path $P_{1}$ from $v_{1}$ to $v_{2}$ contains $\left|a_{1}\right|$ edges with cost function $c_{1}$, the directed path $P_{2}$ from $v_{3}$ to $v_{4}$ contains $a_{2}$ edges with cost function $c_{2}$, the directed path $P_{3}$ from $v_{5}$ to $v_{6}$ contains $a_{2}$ edges with cost function $c_{2}$, and the directed path $P_{4}$ from $v_{7}$ to $v_{8}$ contains $\left|a_{1}\right|$ edges with cost function $c_{1}$. All other edges have an arbitrary cost function in $\mathscr{C}$, say $c_{1}$. Because all costs are strictly positive, for player 1 all strategies except the upper path $P_{u}=\left\{s_{1} \rightarrow v_{1}, P_{1}, v_{2} \rightarrow v_{3}, P_{2}, v_{4} \rightarrow t_{1}\right\}$ and the lower path $P_{d}=\left\{s_{1} \rightarrow v_{5}, P_{3}, v_{6} \rightarrow v_{7}, P_{4}, v_{8} \rightarrow t_{1}\right\}$ are strictly dominated in the sense that they have strictly higher costs than either $P_{u}$ or $P_{d}$ regardless of the strategy played by player 2. For player 2, all strategies except the left path $P_{l}=\left\{s_{2} \rightarrow v_{1}, P_{1}, v_{2} \rightarrow v_{5}, P_{3}, v_{6} \rightarrow t_{2}\right\}$ and the right path $P_{r}=\left\{s_{2} \rightarrow v_{3}, P_{2}, v_{4} \rightarrow v_{7}, P_{4}, v_{8} \rightarrow t_{2}\right\}$ are strictly dominated. We consider the 4-cycle $\gamma=\left(\left(P_{u}, P_{l}\right),\left(P_{d}, P_{l}\right),\left(P_{d}, P_{r}\right),\left(P_{u}, P_{r}\right),\left(P_{u}, P_{l}\right)\right)$, and calculate that

$$
\begin{aligned}
\pi_{1}\left(P_{d}, P_{l}\right)-\pi_{1}\left(P_{u}, P_{l}\right)= & (y-x)\left(c_{1}(y-x)+a_{2} c_{2}(y)+c_{1}(y-x)-a_{1} c_{1}(y-x)+c_{1}(y-x)\right. \\
& \left.\quad-c_{1}(y-x)+a_{1} c_{1}(y)-c_{1}(y-x)-a_{2} c_{2}(y-x)-c_{1}(y-x)\right) \\
= & (y-x)\left(a_{1} c_{1}(y)+a_{2} c_{2}(y)-a_{1} c_{1}(y-x)-a_{2} c_{2}(y-x)\right) \\
= & (y-x)(c(y)-c(y-x)) .
\end{aligned}
$$

In the same fashion, we obtain $\pi_{2}\left(P_{d}, P_{r}\right)-\pi_{2}\left(P_{d}, P_{l}\right)=x(c(x)-c(y)), \pi_{1}\left(P_{u}, P_{r}\right)-\pi_{1}\left(P_{d}, P_{r}\right)=(y-x)$. $(c(y)-c(y-x))$, and $\pi_{2}\left(P_{u}, P_{l}\right)-\pi_{2}\left(P_{u}, P_{r}\right)=x(c(x)-c(y))$. If $c(y-x)>c(y)>c(x)$, then $\gamma$ is an improvement cycle which gives that none of the strategy profiles contained in $\gamma$ is a PNE. If, on the other hand, $c(y-x)<c(y)<c(x)$, we can reverse the direction of $\gamma$ and get an improvement cycle. Because every strategy profile that uses only nondominated strategies is contained in $\gamma$, the constructed directed network congestion game does not admit a PNE.

Second case: $a_{1}>0$ : Consider the network shown in Figure 3(b). Here, both players want to route from $s$ to $t$; that is, $S_{1}=S_{2}=\left\{P_{1}^{\prime}, P_{2}^{\prime}\right\}$. The directed paths $P_{1}^{\prime}$ and $P_{2}^{\prime}$ each contain $a_{1}$ edges with cost function $c_{1}$ and $a_{2}$ edges with cost function $c_{2}$. If $c(y-x)<c(y)<c(x)$, player 1 prefers to be alone on an $(s, t)$-path while player 2 wants to share the path with player 1 . If $c(y-x)>c(y)>c(x)$, the argumentation works the other way round. We conclude that the game does not admit a PNE.

Together with Lemma 4.1 and Theorem 4.1, we obtain the following characterization of consistency for two-player network congestion games on directed networks.

THEOREM 6.1. Let $\mathscr{C}$ be a set of strictly positive and continuous functions and let $\mathscr{G}_{d n}^{2}(\mathscr{C})$ be the set of twoplayer directed network games such that cost functions are in $\mathscr{C}$. Then, the following conditions are equivalent.

(i) $\mathscr{C}$ is consistent for $\mathscr{G}_{d n}^{2}(\mathscr{C})$.

(ii) $\mathscr{C}$ is FIP-consistent for $\mathscr{G}_{d n}^{2}(\mathscr{C})$.

(iii) $\mathscr{C}$ contains only monotonic functions and for all nonconstant $c_{1}, c_{2} \in \mathscr{C}$, there are constants $a, b \in \mathbb{R}$ with $c_{1}(x)=a c_{2}(x)+b$ for all $x \in \mathbb{R}_{\geq 0}$.

Using similar ideas as in the case of two players, we can also prove a version of the Extended Monotonicity Lemma for directed network games with three or more players.

Lemma 6.2 (Extended Monotonicity Lemma for Directed Networks). Let $\mathscr{C}$ be a set of strictly positive and continuous functions. If $\mathscr{C}$ is consistent for three-player directed network congestion games, then $\mathscr{L}_{\mathbb{Z}}^{3}(\mathscr{C})$ contains only monotonic functions.

Proof. Assume by contradiction that there are $a_{1}, a_{2} \in \mathbb{Z}, \delta \in \mathbb{R}_{>0}$ and a monotonic function $c_{1} \in \mathscr{C}$ such that the function $c: \mathbb{R}_{\geq 0} \rightarrow \mathbb{R}$ defined as $c(x)=a_{1} c_{1}(x)+a_{2} c_{1}(x+\delta)$ is not monotonic. We may again assume w.l.o.g. that $c_{1}$ is monotonic and that $a_{2}>0$. Note that because $c_{1}$ is monotonic, this implies $a_{1}<0$.

Consider the network in Figure 4 where again the directed paths $P_{1}$ and $P_{4}$ contain $\left|a_{1}\right|$ edges each, and the directed paths $P_{2}$ and $P_{3}$ contains $a_{2}$ edges each. In addition to the players $i=1,2$ corresponding to the pairs $\left(s_{i}, t_{i}\right), i=1,2$, we now have a third player corresponding to the pair $\left(s_{3}, t_{3}\right)$ with a single strategy $P_{Q}=\left\{P_{3}, Q, P_{2}\right\}$ and demand $d_{3}=\delta$. Moreover, we set $d_{1}=y-x, d_{2}=x$ for $x, y \in \mathbb{R}_{>0}$ with $y>x$ such that either $c(y-x)<c(y)<c(x)$ or $c(y-x)>c(y)>c(x)$ holds (by Lemma 3.2 such values exist). We design the directed path $Q$ from $v_{6}$ to $v_{3}$ so as to contain a sufficiently large number of edges, such that for players 1 and 2 , all $\left(s_{i}, t_{i}\right)$-paths not containing $Q$ are strictly less costly than every path that contains $Q$. As every 


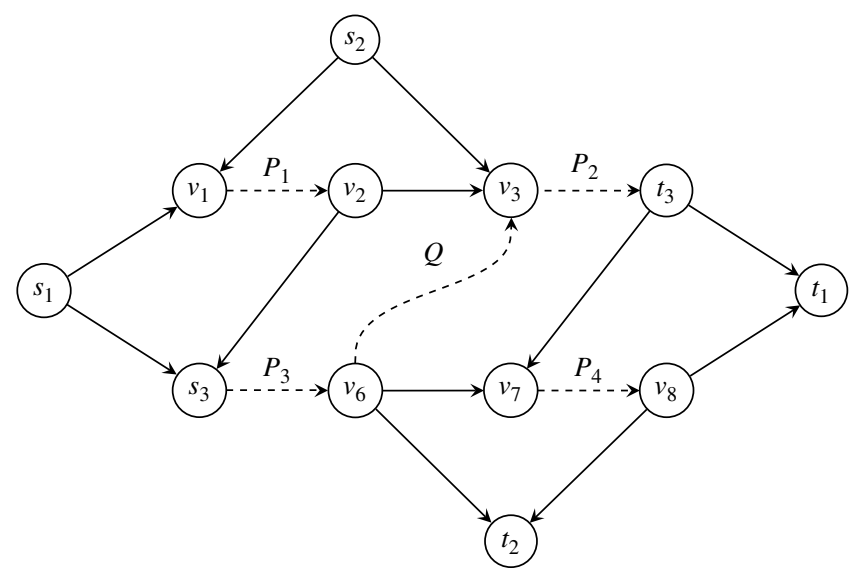

FIGURE 4. Multi-commodity directed network instance used in the proof of the Extended Monotonicity Lemma for Directed Networks (Lemma 6.2).

$\left(s_{i}, t_{i}\right)$-path that does not contain $Q$ has costs less than $2\left(a_{2}-a_{1}+6\right) c_{1}(y+\delta)$ and every edge in $Q$ has cost at least $c_{1}(\delta)$, it is sufficient to let $Q$ contain $2\left(a_{2}-a_{1}+6\right)\left\lceil\left(c_{1}(y+\delta)\right) / c_{1}(\delta)\right\rceil+1$ edges. By construction of $Q$, for player 1, all strategies except the upper path $P_{u}=\left\{s_{1} \rightarrow v_{1}, P_{1}, v_{2} \rightarrow v_{3}, P_{2}, t_{3} \rightarrow t_{1}\right\}$ and the lower path $P_{d}=\left\{s_{1} \rightarrow s_{3}, P_{3}, v_{6} \rightarrow v_{7}, P_{4}, v_{8} \rightarrow t_{1}\right\}$ are strictly dominated in the sense that they have strictly higher costs than either $P_{u}$ or $P_{d}$ regardless of the strategies played by players 2 and 3. For player 2, all strategies except the left path $P_{l}=\left\{s_{2} \rightarrow v_{1}, P_{1}, v_{2} \rightarrow s_{3}, P_{3}, v_{6} \rightarrow t_{2}\right\}$ and the right path $P_{r}=\left\{s_{2} \rightarrow v_{3}, P_{2}, t_{3} \rightarrow v_{7}, P_{4}, v_{8} \rightarrow t_{2}\right\}$ are strictly dominated. With the same calculations as in Lemma 6.1 one can show that the 4-cycle $\gamma=$ $\left(\left(P_{u}, P_{l}, P_{Q}\right),\left(P_{d}, P_{l}, P_{Q}\right),\left(P_{d}, P_{r}, P_{Q}\right),\left(P_{u}, P_{r}, P_{Q}\right),\left(P_{u}, P_{l}, P_{Q}\right)\right)$ is an improvement cycle when traversed in the right direction. Because every strategy profile that uses only nondominated strategies is contained in $\gamma$, we conclude that the thus constructed network congestion game does not admit a PNE.

Using Lemma 5.1, we obtain the following characterization of cost functions that are consistent for weighted directed network congestion games.

THEOREM 6.2. Let $\mathscr{C}$ be a set of strictly positive and continuous functions and let $\mathscr{G}_{d n}(\mathscr{C})$ be the set of directed network congestion games such that cost functions are in $\mathscr{C}$. Then, the following are equivalent:

(i) $\mathscr{C}$ is consistent for $\mathscr{G}_{d n}(\mathscr{C})$.

(ii) $\mathscr{C}$ is FIP-consistent for $\mathscr{G}_{d n}(\mathscr{C})$.

(iii) $\mathscr{C}$ contains only affine functions or $\mathscr{C}$ contains only functions of type $c(x)=a_{c} e^{\phi x}+b_{c}$, where $a_{c}, b_{c} \in \mathbb{R}$ may depend on $c$ while $\phi \in \mathbb{R}$ is independent of $c$.

This characterization is even valid for three-player games.

REMARK 6.1. In games with negative costs the players strive to establish long paths. In this case, our construction does not work since, e.g., player 2 prefers to take the detour $v_{6} \rightarrow v_{7} \rightarrow v_{8} \rightarrow t_{2}$ instead of the edge $v_{6} \rightarrow t_{2}$.

6.2. Undirected networks. We first show that a version of the Extended Monotonicity Lemma holds also for two-player games on undirected networks. In such a game, we are given an undirected graph and for each player $i$, two designated vertices $s_{i}$ and $t_{i}$. Facilities correspond to the edges of the graph and the strategy set of each player $i$ contains all simple $s_{i}, t_{i}$-paths. Each edge can be traversed in any direction and its cost depends on the aggregated flow.

Lemma 6.3 (Extended Monotonicity Lemma for Two-Player Games on Undirected Networks). Let $\mathscr{C}$ be a set of strictly positive and continuous functions. If $\mathscr{C}$ is consistent for two-player undirected network congestion games, then $\mathscr{L}_{\mathbb{Z}}^{2}(\mathscr{C})$ contains only monotonic functions.

Proof. For a contradiction, let $a_{1}, a_{2} \in \mathbb{Z}$ and $c_{1}, c_{2} \in \mathscr{C}$ be such that the function $c: \mathbb{R}_{\geq 0} \rightarrow \mathbb{R}$ defined as $c(x)=a_{1} c_{1}(x)+a_{2} c_{2}(x)$ is not monotonic and w.l.o.g. $a_{2}>0$. Moreover, let $x, y \in \mathbb{R}_{>0}$ with $y>x$ be such that either $c(y-x)<c(y)<c(x)$ or $c(y-x)>c(y)>c(x)$ holds. We set $d_{1}=y-x, d_{2}=x$ and distinguish the following two cases. If $a_{1}<0$ we consider the network in Figure 5 where the paths $P_{1}$ and $P_{4}$ each contain $\left|a_{1}\right|$ edges with cost function $c_{1}$ and the paths $P_{2}$ and $P_{3}$ each contain $a_{2}$ edges with cost function $c_{2}$. With similar calculations as in the proof of Lemma 6.1 one can verify that the 4-cycle $\gamma=\left(\left(P_{1} \cup P_{2}, P_{1} \cup P_{3}\right)\right.$, 

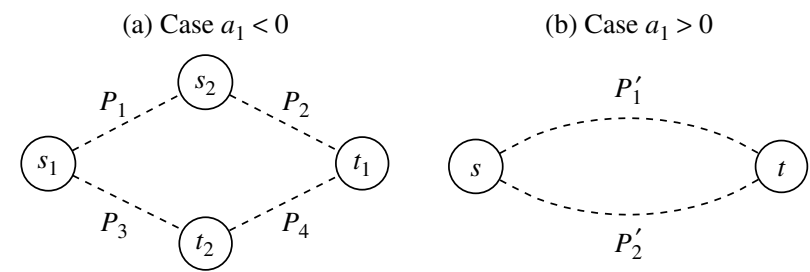

FIGURE 5. Undirected network congestion games used in the proof of the Extended Monotonicity Lemma for Two-Player Undirected Networks (Lemma 6.3).

$\left.\left(P_{3} \cup P_{4}, P_{1} \cup P_{3}\right),\left(P_{3} \cup P_{4}, P_{2} \cup P_{4}\right),\left(P_{1} \cup P_{2}, P_{2} \cup P_{4}\right),\left(P_{1} \cup P_{2}, P_{1} \cup P_{3}\right)\right)$ is an improvement cycle if traversed in the right sense. If on the other hand $a_{1}>0$, we consider the undirected network shown in Figure 5 and obtain the same contradiction as in Lemma 6.1.

Likewise, we obtain the following characterization for two-player games on undirected networks.

THEOREM 6.3. Let $\mathscr{C}$ be a nonempty set of strictly positive and continuous functions and let $\mathscr{G}_{\text {un }}^{2}(\mathscr{C})$ be the set of two-player undirected network games such that cost functions are in $\mathscr{C}$. Then, the following conditions are equivalent.

(i) $\mathscr{C}$ is consistent for $\mathscr{G}_{u n}^{2}(\mathscr{C})$.

(ii) $\mathscr{C}$ is FIP-consistent for $\mathscr{G}_{u n}^{2}(\mathscr{C})$.

(iii) $\mathscr{C}$ contains only monotonic functions and for all nonconstant $c_{1}, c_{2} \in \mathscr{C}$, there are constants $a, b \in \mathbb{R}$ with $c_{1}(x)=a c_{2}(x)+b$ for all $x \in \mathbb{R}_{\geq 0}$.

Turning to games with three players we are not able to characterize the set of consistent cost functions. However, we can still characterize consistency for games with at least four players.

Lemma 6.4 (Extended Monotonicity Lemma for Undirected Networks). Let $\mathscr{b}$ be a set of strictly positive and continuous functions. If $\mathscr{C}$ is consistent for undirected network congestion games with at least four players, then $\mathscr{L}_{\mathbb{Z}}^{3}(\mathscr{C})$ contains only monotonic functions.

Proof. For a contradiction, suppose that there are $a_{1}, a_{2} \in \mathbb{Z}, \delta \in \mathbb{R}_{>0}$, and a monotonic function $c_{1} \in \mathscr{C}$ such that the function $c: \mathbb{R}_{\geq 0} \rightarrow \mathbb{R}$ defined as $c(x)=a_{1} c_{1}(x)+a_{2} c_{1}(x+\delta)$ is not monotonic; again w.l.o.g., $c_{1}$ is monotonic, $a_{2}>0$ and $a_{1}<0$.

Consider the network in Figure 6 where the paths $P_{1}$ and $P_{4}$ each contain $\left|a_{1}\right|$ edges and the paths $P_{2}$ and $P_{3}$ each contain $a_{2}$ edges. The players $i=1,2$ correspond to the source sink pairs $\left(s_{i}, t_{i}\right), i=1,2$. Additionally, there are players associated with the source sink pairs $\left(s_{i}, t_{i}\right), i=3,4$, and demand $d_{3}=d_{4}=\delta$. Moreover, we set $d_{1}=y-x, d_{2}=x$ for $x, y \in \mathbb{R}_{>0}$ with $y>x$ such that either $c(y-x)<c(y)<c(x)$ or $c(y-x)>c(y)>c(x)$ holds (by Lemma 3.2 such values exist).

We endow every edge in the paths $Q_{1}, \ldots, Q_{8}$ with cost function $c_{2}$ and make them sufficiently long such that players 3 and 4 always prefer to choose a strategy not containing any of these paths. Because the paths $P_{2}$ and $P_{3}$ have costs less than $a_{2} c_{2}(y+2 \delta)$, and every edge in $Q_{i}, i=1, \ldots, 8$ used by players 3 or 4 has cost

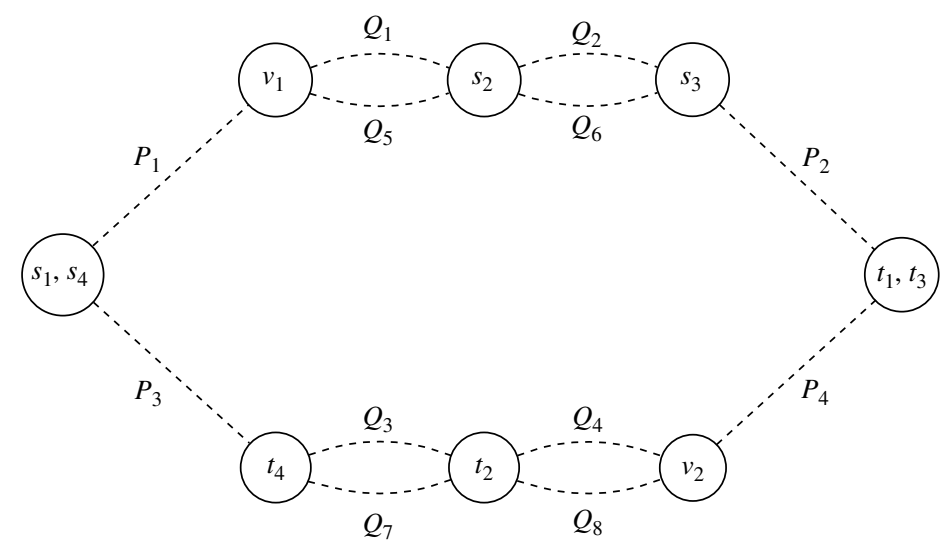

FIGURE 6. Undirected network congestion games used in the proof of the Extended Monotonicity Lemma for Undirected Networks (Lemma 6.4). 
at least $c_{2}(\delta)$, it suffices for all $i=1, \ldots, 8$ to let $Q_{i}$ contain $a_{2}\left\lceil\left(c_{2}(y+2 \delta)\right) / c_{2}(\delta)\right\rceil+1$ edges each. Then, for player 3, all strategies except $P_{2}$ are strictly dominated by $P_{2}$ and for player 4 all strategies except $P_{3}$ are strictly dominated by $P_{3}$. Given that players 3 and 4 will not use any of the $Q_{i}$-paths in equilibrium, we may assume that players 1 and 2 will not share any of the $Q_{i}$ paths in equilibrium; w.l.o.g. player 1 always uses the paths $Q_{1}, \ldots, Q_{4}$ instead of $Q_{5}, \ldots, Q_{8}$ while player 2 always uses paths $Q_{5}, \ldots, Q_{8}$ instead of $Q_{1}, \ldots, Q_{4}$. With the same calculations as before one can show that there is an improvement cycle $\gamma$ of the form $\gamma=$ $\left(\left(P_{u}, P_{l}, P_{2}, P_{3}\right),\left(P_{d}, P_{l}, P_{2}, P_{3}\right),\left(P_{d}, P_{r}, P_{2}, P_{3}\right),\left(P_{u}, P_{r}, P_{2}, P_{3}\right),\left(P_{u}, P_{l}, P_{2}, P_{3}\right)\right)$, where $P_{u}=P_{1} \cup Q_{1} \cup Q_{2} \cup P_{2}$, $P_{d}=P_{3} \cup Q_{3} \cup Q_{4} \cup P_{4}, P_{l}=Q_{5} \cup P_{1} \cup P_{3} \cup Q_{7}$, and $P_{r}=Q_{6} \cup P_{2} \cup Q_{4} \cup Q_{8}$. Because every strategy profile that uses only nondominated strategies is contained in $\gamma$, the thus constructed network congestion game does not admit a PNE.

Using the above lemma, we obtain the following result.

THEOREM 6.4. Let $\mathscr{C}$ be a set of strictly positive and continuous functions and let $\mathscr{G}_{\text {un }}(\mathscr{C})$ be the set of undirected network congestion games with at least four players and cost functions in $\mathscr{C}$. Then, the following are equivalent:

(i) $\mathscr{C}$ is consistent for $\mathscr{G}_{\text {un }}(\mathscr{C})$.

(ii) $\mathscr{C}$ is FIP-consistent for $\mathscr{G}_{u n}(\mathscr{C})$.

(iii) $\mathscr{C}$ contains only affine functions or $\mathscr{C}$ contains only functions of type $c(x)=a_{c} e^{\phi x}+b_{c}$, where $a_{c}, b_{c} \in \mathbb{R}$ may depend on $c$ while $\phi \in \mathbb{R}$ is independent of $c$.

For single-commodity network games (directed or undirected) we are not able to characterize consistency of cost functions. However, by introducing a super-source and a super-sink to the network constructions used, it follows that the improvement cycles are preserved; thus, all characterizations for FIP-consistency obtained in this section continue to hold.

7. Weighted singleton congestion games. In this section, we consider the case of singleton weighted congestion games. In this class of games, for every player $i$, every strategy $s_{i} \in S_{i}$ contains a single facility only. As mentioned in Corollary 3.1, the construction of the Monotonicity Lemma (Lemma 3.3) is even valid for singleton games, establishing that every set of continuous cost functions $\mathscr{C}$ that is consistent for singleton games may only contain monotonic functions. It is well known that singleton congestion games with weighted players and either only nondecreasing or only nonincreasing cost functions admit a PNE; see Even-Dar et al. [14], Fotakis et al. [15], and Rozenfeld and Tennenholtz [36]. Since the positive result for nondecreasing costs is established via a potential function, these games also possess the FIP. With similar arguments it is not difficult to establish the FIP also for the case of nonincreasing costs. ${ }^{1}$ To the best of our knowledge it was not known before whether singleton weighted congestion games with both nondecreasing and nonincreasing cost functions admit a PNE or even the FIP. Regarding the existence of PNE, for two-player games, we give a positive answer to this question.

THeORem 7.1. Let $\mathscr{C}$ be a set of continuous functions and let $\mathscr{G}_{s g l}^{2}(\mathscr{C})$ be the set of two-player games such that cost functions are in $\mathscr{C}$ and strategy spaces are sets of singletons. Then, $\mathscr{C}$ is consistent for $\mathscr{G}_{s g l}^{2}(\mathscr{C})$ if and only if $\mathscr{C}$ contains only monotonic functions.

Proof. The "only if" part follows from Corollary 3.1. For the "if" part let $M=\left(N, F, S,\left(c_{f}\right)_{f \in F}\right)$ be a congestion model with $|N|=2$. W.l.o.g. we assume $d_{1} \leq d_{2}$. We partition the set of facilities into sets $F_{-}$and $F_{+}$, where $F_{+}$contains all facilities with nondecreasing cost functions (including all facilities with constant functions) and $F_{-}$all other facilities. W.l.o.g. we can assume that both players have access to all facilities in $F_{-}$, since we can replace the cost function of every facility that is contained in the strategy space of only one player by a constant function. We initialize the players both playing $g$, where $g=\arg \min _{f \in F_{-}} c_{f}\left(d_{1}+d_{2}\right)$. We distinguish two cases.

First case: Player 1 has an improving move from $(\{g\},\{g\})$. In this case, we let player 1 move to one of her best replies $\left\{f_{1}\right\} \in S_{1}$. Using the special choice of $g$, we have $f_{1} \in F_{+}$. If player 2 does not have an improving move from $\left(\left\{f_{1}\right\},\{g\}\right)$, we are done. So, let $\left\{f_{2}\right\}$ be a best reply of player 2 to $\left(\left\{f_{1}\right\},\{g\}\right)$. If $f_{1} \neq f_{2}$, we claim that $\left(\left\{f_{1}\right\},\left\{f_{2}\right\}\right)$ is a PNE. To see this, note that if $f_{2} \in F_{+}$, then player 2 switching from $\{g\}$ to $\left\{f_{2}\right\}$ does not make any of the facilities more attractive to player 1 . If on the other hand, $f_{2} \in F_{-}$, we get $\pi_{1}\left(\left\{f_{2}\right\},\left\{f_{2}\right\}\right) \geq \pi_{1}(\{g\},\{g\})>\pi_{1}\left(\left\{f_{1}\right\},\left\{f_{2}\right\}\right)=\pi_{1}\left(\left\{f_{1}\right\},\{g\}\right)$ by the choice of $g$; thus player 1 does not want

${ }^{1}$ Consider the function $\phi$ that assigns to each strategy profile the nondecreasingly sorted vector of the scaled players' private costs $\left(\pi_{i} / d_{i}\right)_{i \in N}$. Then, $\phi$ decreases lexicographically along any improvement path, establishing that every such path is finite. 
Harks and Klimm: Pure Nash Equilibria in Weighted Congestion Games

TABle 1. (a) Cost functions of the five facilities $g, f_{1}, f_{2}, f_{3}$, and $f_{4}$ in the game of Example 7.1; (b) Cost functions of the three facilities $f, g$, and $h$ in the game of Example 7.2.

(a) Cost functions in the game of Example 7.1

\begin{tabular}{lccc}
\hline & \multicolumn{3}{c}{ Cost $c(x)$} \\
\cline { 2 - 4 } Facility & $x=1$ & $x=2$ & $x=3$ \\
\hline$g$ & 10 & 5 & 3 \\
$f_{1}$ & 2 & 2 & 9 \\
$f_{2}$ & 8 & 8 & 8 \\
$f_{3}$ & 1 & 7 & 7 \\
$f_{4}$ & 6 & 6 & 6
\end{tabular}

(b) Cost functions in the game of Example 7.2

\begin{tabular}{lcccccc}
\hline & \multicolumn{5}{c}{ Cost $c(x)$} \\
\cline { 2 - 6 } Facility & $x=1$ & $x=2$ & $x=3$ & $x=4$ & $x=5$ & $x=6$ \\
\hline$f$ & 0 & 0 & 2 & 3 & 3 & 3 \\
$g$ & 5 & 1 & 1 & 1 & 0 & 0 \\
$h$ & 2 & 2 & 2 & 2 & 4 & 4 \\
\hline
\end{tabular}

to move to $f_{2}$ and we have reached an equilibrium. The only interesting case that remains is $\left\{f_{1}\right\}=\left\{f_{2}\right\}$. Again, if player 1 does not have an improving move, there is nothing left to show, so let $\left\{h_{1}\right\} \neq\left\{f_{1}\right\}$ be a best reply of player 1 to $\left(\left\{f_{1}\right\},\left\{f_{1}\right\}\right)$. Note that $h_{1} \notin F_{-}$because otherwise we get $\pi_{2}\left(\left\{f_{1}\right\},\left\{h_{1}\right\}\right) / d_{2} \leq \pi_{1}\left(\left\{h_{1}\right\},\left\{f_{1}\right\}\right) / d_{1}<$ $\pi_{1}\left(\left\{f_{1}\right\},\left\{f_{1}\right\}\right) / d_{1}=\pi_{2}\left(\left\{f_{1}\right\},\left\{f_{1}\right\}\right) / d_{2}$, where the first inequality follows since $d_{2} \geq d_{1}$. This is a contradiction to the fact that $\left\{f_{1}\right\}$ was a best reply of player 2 . As $\pi_{2}\left(\left\{h_{1}\right\},\left\{f_{1}\right\}\right) \leq \pi_{2}\left(\left\{f_{1}\right\},\left\{f_{1}\right\}\right)$, player 2 does not want to deviate from $\left(\left\{h_{1}\right\},\left\{f_{2}\right\}\right)$. Also, player 1 will not deviate from $\left(\left\{h_{1}\right\},\left\{f_{2}\right\}\right)$ as $\left\{h_{1}\right\}$ was a best reply.

Second case: Player 1 has no improving move from $(\{g\},\{g\})$. If, also, player 2 does not have an improving move from $(\{g\},\{g\})$, we are done. Otherwise, let $\left\{f_{2}\right\} \in S_{2}$ be a best reply of player 2 . Note that $\left\{f_{2}\right\} \notin S_{1}$ because otherwise $\left\{f_{2}\right\}$ would have been an improving move from $(\{g\},\{g\})$ of player 1 . If player 1 has no improving move from $\left(\{g\},\left\{f_{2}\right\}\right)$, we are done. Otherwise let $\left\{f_{1}\right\}$ be a best reply of player 1 to $\left(\{g\},\left\{f_{2}\right\}\right)$. Using that $f_{1} \neq f_{2}$ and that $\pi_{2}\left(\left\{f_{1}\right\},\left\{f_{2}\right\}\right) \leq \pi_{2}(\{g\},\{g\})$, we have that $\left(\left\{f_{1}\right\},\left\{f_{2}\right\}\right)$ is a PNE.

Two-player singleton weighted congestion games with monotonic costs need not possess the FIP as shown in the following example.

Example 7.1. Consider the congestion model $M=\left(N, F, S,\left(c_{f}\right)_{f \in F}\right)$ with two players $N=\{1,2\}$ who have access to all five facilities $F=\left\{g, f_{1}, f_{2}, f_{3}, f_{4}\right\}$. The cost functions of the facilities are shown in Table 1(a). Note that the cost function of facility $g$ is strictly decreasing while all other cost functions are nondecreasing. The players' demands are given by $d_{1}=1$ and $d_{2}=2$. It is not hard to verify that the cycle $\gamma$ defined as $\gamma=$ $\left((\{g\},\{g\}),\left(\{g\},\left\{f_{1}\right\}\right),\left(\left\{f_{1}\right\},\left\{f_{1}\right\}\right),\left(\left\{f_{1}\right\},\left\{f_{2}\right\}\right),\left(\left\{f_{3}\right\},\left\{f_{2}\right\}\right),\left(\left\{f_{3}\right\},\left\{f_{3}\right\}\right),\left(\left\{f_{4}\right\},\left\{f_{3}\right\}\right),\left(\left\{f_{4}\right\},\{g\}\right),(\{g\},\{g\})\right)$ is an improvement cycle.

We proceed to show that for singleton games with three players monotonicity of cost functions alone is not enough for the existence of a PNE. This is illustrated in the following example.

Example 7.2. Consider the congestion model $M=\left(N, F, S,\left(c_{f}\right)_{f \in F}\right)$ with $N=\{1,2,3\}$ and $F=\{f, g, h\}$. The used cost functions are given in Table 1(b). We claim that the weighted congestion game $G(\mathcal{M})=(N, S, \pi)$ with $S_{1}=\{\{g\},\{h\}\}, S_{2}=\{\{f\},\{g\}\}, S_{3}=\{\{f\},\{h\}\}$ and $d_{1}=1, d_{2}=2, d_{3}=4$ does not admit a PNE. To see

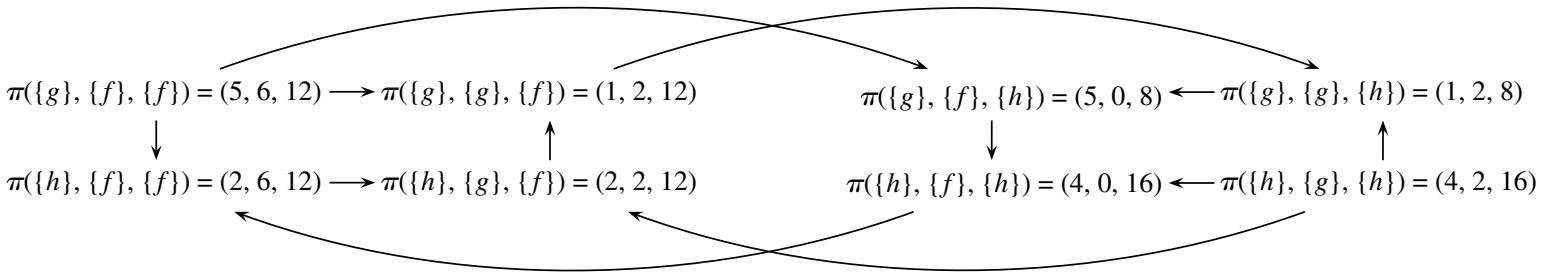

FIGURE 7. Best reply graph of the singleton weighted congestion game $G(M)$ constructed in Example 7.2.

Notes. The vertical arcs correspond to best replies of player 1, the straight horizontal arcs to best replies of player 2 and the wide horizontal arcs to best replies of player 3. Since the graph does not have a sink, the game $G(\mathcal{M})$ does not possess a PNE. 
this, note that the best-reply graph $\gamma$ shown in Figure 7 does not have a sink. Using that all strategy profiles are contained in $\gamma$ the claimed result follows.

However, we are able to give a positive result for symmetric games in which the players have access to all facilities.

THEOREM 7.2. Let $\mathscr{C}$ be a set of continuous functions and let $\mathscr{G}_{\text {sgl,sym }}(\mathscr{C})$ be the set of games such that cost functions are in $\mathscr{b}$ and strategy spaces are sets of singletons and equal for every player. Then, $\mathscr{C}$ is consistent for $\mathscr{G}_{\text {sgl,sym }}(\mathscr{C})$ if and only if $\mathscr{C}$ contains only monotonic functions.

Note that the only if part also follows from Corollary 3.1. To prove the if part, we give an algorithm that efficiently computes a PNE in such games. In the following, we denote by $F_{+}$and $F_{-}$the set of facilities with nondecreasing and nonincreasing costs, respectively. To obtain a partition of $F$, we introduce the convention that facilities with constant cost functions are contained in $F_{+}$only. The algorithm that we propose (Algorithm 1) initializes all players on the facility $g \in F_{-}$that minimizes $c_{g}\left(\sum_{i \in N} d_{i}\right)$. Clearly then, no player has an incentive to switch to another facility $h \in F_{-}$. The key observation is that, as long as there is at least one player $i \in N$ that wants to switch to a facility $f \in F_{+}$, the player with smallest demand also does so. So we iteratively take the player with smallest weight on $g$ and let her move to $F_{+}$. Then, we compute a sequence of best replies of the players on $F_{+}$in order to assure that none of them has an incentive to deviate to another facility in $F_{+}$. Also, the players on $F_{-}$are placed on the facility minimizing $c_{f}\left(\sum_{i \in N: s_{i} \in F_{-}} d_{i}\right)$. Since we can prove that a player on $F_{+}$ never wants to move back to a facility in $F_{-}$, this process stops after a polynomial number of best-reply steps.

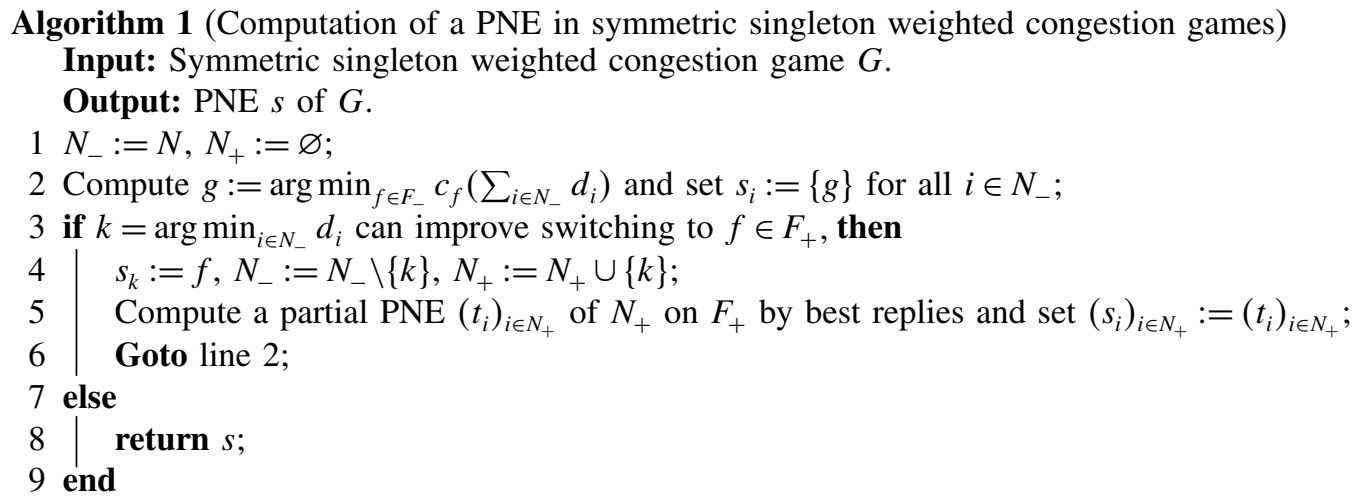

\section{LEMma 7.1. Algorithm 1 computes a PNE.}

Proof. Let us first remark that the computation of the partial PNE of players $N_{+}$on $F_{+}$in line 5 finishes after a finite sequence of best replies since the cost functions of the facilities in $F_{+}$are nondecreasing; see Ackermann et al. [1], and Ieong et al. [23]. As at most $n$ times such PNE is computed, the algorithm terminates after a finite number of best-reply steps. Let $z$ denote the outcome of the algorithm. Clearly, no player $j \in N_{+}$can improve switching to another facility $f \in F_{+}$since we always recompute a partial PNE in line 5. Also, no player $j \in N_{-}$can improve unilaterally deviating to another facility $f \in F_{-}$since $c_{f}\left(d_{j}\right) \geq c_{f}\left(\sum_{i \in N_{-}} d_{i}\right) \geq c_{g}\left(\sum_{i \in N_{-}} d_{i}\right)$. In addition, we know that player $k=\arg \min _{i \in N} d_{i}$ does not improve switching from facility $g$ to another facility $f \in F_{+}$. In consequence, the same holds for every other player $j \in N_{-}$since the costs for her on a facility $f \in F_{+}$are not smaller. Finally, it is left to show that in $z$ no player $j \in N_{+}$has an interest in switching to some facility $f \in F_{-}$.

To prove this, let $i_{t}, t=1, \ldots, T, T \in \mathbb{N}$, denote the player that switches from $g_{t} \in F_{-}$to $f_{t} \in F_{+}$in the $t$ th iteration of the algorithm and let $\tilde{z}^{t}$ and $z^{t}$ denote the corresponding strategy profiles before and after the re-computation of the partial PNE on $F_{+}$in line 5, respectively. We claim that

$$
\min _{g \in F_{-}} c_{g}\left(\ell_{g}\left(z^{t}\right)+d_{i_{t}}\right)>\max _{f \in F_{+}: \ell_{f}\left(z^{t}\right)>0} c_{f}\left(\ell_{f}\left(z^{t}\right)\right) \text { for all } t=1, \ldots, T,
$$

where $\ell_{g}\left(z^{t}\right)$ and $\ell_{f}\left(z^{t}\right)$ denote the load on facility $g$ (respectively, $f$ ) in strategy profile $z^{t}$. For $t=$ 1 , the statement holds true, since player $i_{1}$ improves switching from $F_{-}$to $F_{+}$. Now, suppose (9) holds true for $t-1$. In the $t$ th iteration, player $i_{t}$ changes her strategy from $g_{t} \in F_{-}$to some facility $f_{t} \in F_{+}$. In consequence, $\min _{g \in F_{-}} c_{g}\left(\ell_{g}\left(z^{t}\right)+d_{i_{t}}\right)=c_{g_{t}}\left(\ell_{g_{t}}\left(z^{t}\right)+d_{i_{t}}\right)>c_{f_{t}}\left(\ell_{f_{t}}\left(\tilde{z}^{t}\right)\right)$. As the facilities in $F_{-}$have nonincreasing cost functions, we obtain $\min _{g \in F_{-}} c_{g}\left(\ell_{g}\left(z^{t}\right)+d_{i_{t}}\right) \geq \min _{g \in F_{-}} c_{g}\left(\ell_{g}\left(z^{t-1}\right)+d_{i_{t-1}}\right)$. By the induction hypothesis, this implies $\min _{g \in F_{-}} c_{g}\left(\ell_{g}\left(z^{t}\right)+d_{i_{t}}\right)>c_{f}\left(\ell_{f}\left(\tilde{z}^{t}\right)\right)$ for all $f \in F_{+} \backslash\left\{f_{t}\right\}$ with $\ell_{f}\left(\tilde{z}^{t}\right)>0$. Thus, we 
have established $\min _{g \in F_{-}} c_{g}\left(\ell_{g}\left(z^{t}\right)+d_{i_{t}}\right)>\max _{f \in F_{+}: \ell_{f}\left(\tilde{z}^{t}\right)>0} c_{f}\left(\ell_{f}\left(\tilde{z}^{t}\right)\right)$. Since the maximum cost on $F_{+}$cannot increase in the sequence of best-reply steps (c.f. Harks et al. [21]), we obtain $\min _{g \in F_{-}} c_{g}\left(\ell_{g}\left(z^{t}\right)+d_{i_{t}}\right)>$ $\max _{f \in F_{+}: \ell_{f}\left(z^{t}\right)>0} c_{f}\left(\ell_{f}\left(z^{t}\right)\right)$ as claimed.

Because the algorithm always moves the player with the current smallest weight from $F_{-}$to $F_{+}$(line 3 ) it holds that $d_{i_{T}}=\max _{i \in N_{+}} d_{i}$ which gives $\min _{g \in F_{-}} c_{g}\left(\ell_{g}(z)+d_{i}\right) \geq \min _{g \in F_{-}} c_{g}\left(\ell_{g}(z)+d_{i_{T}}\right)>\max _{f \in F_{+}} c_{f}\left(\ell_{f}(\tilde{z})\right)$ for all $i \in N_{+}$. Thus, no player $i \in N_{+}$has an incentive to switch to a facility $g \in F_{-}$.

While the above result implies that the set $\mathscr{C}$ of continuous and monotonic cost functions is consistent for symmetric singleton games, Example 7.1 implies that $\mathscr{C}$ is not FIP-consistent.

8. Conclusions. We obtained a characterization of the equilibrium existence problem in weighted congestion games with respect to the facilities' cost functions. The following issues have not been resolved. Our characterizations for network games require that cost functions are strictly positive. Moreover, for single-commodity games we were only able to characterize the FIP, not consistency. The single-commodity case, however, behaves completely differently as every congestion game with positive and nonincreasing costs admits a PNE in which all players use the socially optimal path (see also Anshelevich et al. [5] for a similar result in the context of network design games). Finally, it would be interesting to characterize consistency of cost functions for undirected networks with three players.

Acknowledgments. We thank Hans-Christian Kreusler for fruitful discussions related to the characterization of continuous and monotonic functions. We are very grateful to the associate editor and the two anonymous referees for their careful proofreading, constructive comments and their discovery of gaps in previous versions of this paper. The research of the second author was supported by the Deutsche Forschungsgemeinschaft within the research training group "Methods for Discrete Structures" (GRK 1408).

\section{References}

[1] Ackermann H, Röglin H, Vöcking B (2009) Pure Nash equilibria in player-specific and weighted congestion games. Theoret. Comput. Sci. 410(17):1552-1563.

[2] Ackermann H, Skopalik A (2007) On the complexity of pure Nash equilibria in player-specific network congestion games. Deng X, Graham F, eds. Proc. 3rd Internat. Workshop on Internet and Network Econom., LNCS, Vol. 4858, 419-430.

[3] Aland S, Dumrauf D, Gairing M, Monien B, Schoppmann F (2006) Exact price of anarchy for polynomial congestion games. Durand B, Thomas W, eds. Proc. 23rd Internat. Sympos. Theoretical Aspects Comput. Sci., LNCS, Vol. 3884, 218-229.

[4] Andelman N, Feldman M, Mansour Y (2009) Strong price of anarchy. Games Econom. Behav. 65(2):289-317.

[5] Anshelevich E, Dasgupta A, Kleinberg J, Tardos É, Wexler T, Roughgarden T (2008) The price of stability for network design with fair cost allocation. SIAM J. Comput. 38(4):1602-1623.

[6] Awerbuch B, Azar Y, Epstein A (2005) The price of routing unsplittable flow. Gabow HN, Fagin R, eds. Proc. 37th Annual ACM Sympos. Theory Comput. (ACM Press), 57-66.

[7] Balakrishnan VK (1996) Introductory Discrete Mathematics (Dover Publications, New York).

[8] Bhaskar U, Fleischer L, Hoy D, Huang C (2009) Equilibria of atomic flow games are not unique. Mathieu C, ed. Proc. 20th Annual ACM-SIAM Sympos. Discrete Algorithms. (SIAM, Philadelphia) 748-757.

[9] Bhawalkar K, Gairing M, Roughgarden T (2010) Weighted congestion games: Price of anarchy, universal worst-case examples, and tightness. de Berg M, Meyer U, eds. Proc. 18th Annual Eur. Sympos. Algorithms, Part II, LNCS, Vol. 6347, 17-28.

[10] Bureau of Public Roads (1964) Traffic assignment manual. U.S. Department of Commerce, Urban Planning Division, Washington, DC.

[11] Chen H-L, Roughgarden T (2009) Network design with weighted players. Theory Comput. Syst. 45(2):302-324.

[12] Christodoulou G, Koutsoupias E (2005) The price of anarchy of finite congestion games. Gabow HN, Fagin R, eds. Proc. 37th Annual ACM Sympos. Theory Comput., 67-73.

[13] Dunkel J, Schulz A (2008) On the complexity of pure-strategy Nash equilibria in congestion and local-effect games. Math. Oper. Res. 33(4):851-868.

[14] Even-Dar E, Kesselman A, Mansour Y (2007) Convergence time to Nash equilibrium in load balancing. ACM Trans. Algorithms 3(3):32.

[15] Fotakis D, Kontogiannis S, Koutsoupias E, Mavronicolas M, Spirakis P (2002) The structure and complexity of Nash equilibria for a selfish routing game. Widmayer P, Triguero F, Morales R, Hennessy M, Eidenbenz S, Conejo R, eds. Proc. 29th Internat. Colloquium on Automata, Languages, and Programming, LNCS, Vol. 2380, 123-134.

[16] Fotakis D, Kontogiannis S, Spirakis P (2005) Selfish unsplittable flows. Theoret. Comput. Sci. 348(2-3):226-239.

[17] Fotakis D, Kontogiannis S, Spirakis P (2006) Atomic congestion games among coalitions. Bugliesi M, Preneel B, Sassone V, Wegener I, eds. Proc. 33rd Internat. Colloquium on Automata, Languages, and Programming, LNCS, Vol. 4052, 572-583.

[18] Gairing M, Monien B, Tiemann K (2006) Routing (un-)splittable flow in games with player-specific linear latency functions. Bugliesi M, Preneel B, Sassone V, Wegener I, eds. Proc. 33rd Internat. Colloquium on Automata, Languages, and Programming, LNCS, Vol. 4051, 501-512.

[19] Goemans M, Mirrokni V, Vetta A (2005) Sink equilibria and convergence. Proc. 46th Annual IEEE Sympos. Foundations Comput. Sci. $142-154$ 
[20] Harks T, Klimm M (2010) On the existence of pure Nash equilibria in weighted congestion games. Abramsky S, Gavoille C, Kirchner C, Meyer auf der Heide F, Spirakis P, eds. Proc. 37th Internat. Colloquium on Automata, Languages, and Programming, LNCS, Vol. 6198, 79-89.

[21] Harks T, Klimm M, Möhring R (2009) Strong Nash equilibria in games with the lexicographical improvement property. Leonardi S, ed. Proc. 5th Internat. Workshop on Internet and Network Econom., LNCS, Vol. 5929, 463-470.

[22] Harks T, Klimm M, Möhring R (2011) Characterizing the existence of potential functions in weighted congestion games. Theory Comput. Systems 49(1):46-70.

[23] Ieong S, McGrew R, Nudelman E, Shoham Y, Sun Q (2005) Fast and compact: A simple class of congestion games. Proc. 20th National Conf. Artificial Intelligence and the 17th Innovative Appl. Artificial Intelligence Conf., 489-494.

[24] Libman L, Orda A (2001) Atomic resource sharing in noncooperative networks. Telecommun. Syst. 17(4):385-409.

[25] Milchtaich I (1996) Congestion games with player-specific payoff functions. Games Econom. Behav. 13(1):111-124.

[26] Milchtaich I (2005) Topological conditions for uniqueness of equilibrium in networks. Math. Oper. Res. 30(1):225-244.

[27] Milchtaich I (2006) The equilibrium existence problem in finite network congestion games. Mavronicolas M, Kontogiannis S, eds. Proc. 2nd Internat. Workshop on Internet and Network Econom., LNCS, Vol. 4286, 87-98.

[28] Monderer D, Shapley L (1996) Potential games. Games Econom. Behav. 14(1):124-143.

[29] Orda A, Rom R, Shimkin N (1993) Competitive routing in multi-user communication networks. IEEE Trans. Networking 1:510-521.

[30] Panagopoulou P, Spirakis P (2006) Algorithms for pure Nash equilibria in weighted congestion games. ACM J. Exp. Algorithmics 11(2.7):1-19.

[31] Richman O, Shimkin N (2007) Topological uniqueness of the Nash equilibrium for selfish routing with atomic users. Math. Oper. Res. 32(1):215-232.

[32] Rosenthal R (1973) A class of games possessing pure-strategy Nash equilibria. Internat. J. Game Theory 2(1) 65-67.

[33] Rosenthal R (1973) The network equilibrium problem in integers. Networks 3(1):53-59.

[34] Roughgarden T (2009) Intrinsic robustness of the price of anarchy. Mitzenmacher M, ed. Proc. 41st Annual ACM Sympos. Theory Comput., 513-522.

[35] Roughgarden T, Tardos É (2002) How bad is selfish routing? J. ACM 49(2):236-259.

[36] Rozenfeld O, Tennenholtz M (2006) Strong and correlated strong equilibria in monotone congestion games. Spirakis P, Mavronicolas M, Kontogiannis S, eds. Proc. 2nd Internat. Workshop on Internet and Network Econom., LNCS, Vol. 4286, 74-86.

[37] Yang H, Zhang X (2008) Existence of anonymous link tolls for system optimum on networks with mixed equilibrium behaviors. Transportation Res. Part B: Methodological 42(2):99-112.

\section{CORRECTION}

In this article, "On the Existence of Pure Nash Equilibria in Weighted Congestion Games" by Tobias Harks and Max Klimm (first published in Articles in Advance May 17, 2012, Mathematics of Operations Research, DOI:10.1287/moor.1120.0543), the following changes were made:

(1) On page 2, Section 1.1, the following sentence was deleted: "Moreover, as a by-product of our analysis, we obtain a polynomial time algorithm computing a PNE for two-player singleton games and symmetric singleton games provided that cost functions are monotonic."

(2) On page 16, Lemma 7.1, the words "in polynomial time" was deleted from the title of the lemma and in the proof, the term "polynomial" was replaced by "finite." It is corrected to read as follows:

“Lemma 7.1. Algorithm 1 computes a PNE.

Proof. Let us first remark that the computation of the partial PNE of players $N_{+}$on $F_{+}$in line 5 finishes after a finite sequence of best replies since the cost functions of the facilities in $F_{+}$are nondecreasing; see Ackermann et al. [1], and Ieong et al. [23]. As at most $n$ times such PNE is computed, the algorithm terminates after a finite number of best-reply steps...." 